Supporting Information (SI) to

\title{
National Empirical Models of Air Pollution Using Microscale Measures of the Urban Environment
}

Tianjun Lu ${ }^{1 *}$, Julian D. Marshall², Wenwen Zhang ${ }^{3}$, Perry Hystad ${ }^{4}$, Sun-Young Kim ${ }^{5}$, Matthew J. Bechle ${ }^{2}$, Matthias Demuzere ${ }^{6}$, Steve Hankey ${ }^{7}$

${ }^{1}$ Department of Earth Science \& Geography, California State University Dominguez Hills, 1000

E. Victoria St, Carson, California 90747, United States

${ }^{2}$ Department of Civil \& Environmental Engineering, University of Washington, 201 More Hall, Seattle, Washington 98195, United States

${ }^{3}$ Edward J. Bloustein School of Planning and Public Policy, Rutgers University, 33 Livingston Ave., New Brunswick, New Jersey 08901, United States

${ }^{4}$ College of Public Health and Human Sciences, Oregon State University, 2520 Campus Way, Corvallis, Oregon 97331, United States

${ }^{5}$ Department of Cancer Control and Population Health, Graduate School of Cancer Science and Policy, National Cancer Center, Goyang-si, Gyeonggi-do, 10408, Korea

${ }^{6}$ Urban Climatology Group, Department of Geography, Ruhr-University Bochum, Bochum, Germany

${ }^{7}$ School of Public and International Affairs, Virginia Tech, 140 Otey Street, Blacksburg, Virginia 24061, United States 


\title{
This document contains
}

\author{
41 pages
}

32 figures

7 tables

Table S1. Candidate geographic predictor variables.

Table S2. Candidate predictor variables of satellite estimates.

Table S3. Categories of POI data.

Table S4. Categories of GSV data.

Table S5. Machine learning hyperparameters.

Table S6. Descriptive statistics of the criteria pollutants.

Table S7. The top 30 variables selected as predictor variables for PLS-K models.

Figure S1. Example 1 of the extracted street view features using the deep learning algorithm.

Figure S2. Example 2 of the extracted street view features using the deep learning algorithm.

Figures S3. LCZ classification.

Figures S4. LCZ surface for the continental US.

Figures S5. LCZ surface for two selected urban areas in the US.

Figures S6. Random 10-fold CV results $\left(\mathrm{R}^{2}\right)$ of the eight $\mathrm{ML}$ algorithms.

Figures S7. Random 10-fold CV results (RMSE) of the eight ML algorithms.

Figures S8. Spatial 10-fold CV results $\left(\mathrm{R}^{2}\right)$ of the eight $\mathrm{ML}$ algorithms.

Figures S9. Spatial 10-fold CV results (RMSE) of the eight ML algorithms.

Figure S10. Comparison of random and spatial CV results.

Figures S11-S17. Variable importance of the top 20 variables by variable type and modeling approach.

Figures S18-S20. Model performance by 10 quantiles.

Figures S21-S23. Model performance by four Census Bureau-designated regions.

Figures S24-S26. Model performance by urban and rural area.

Figures S27-S29. Model performance by demographic index.

Figures S30-S31. Predicted $\mathrm{NO}_{2}$ and $\mathrm{PM}_{2.5}$ concentrations for downtown Blacksburg, VA.

Figure S32. The zoning map of Blacksburg, VA, USA. 
Table S1. Candidate geographic predictor variables

\begin{tabular}{|c|c|c|}
\hline Category & Measure & Note $^{\mathrm{a}}$ \\
\hline Traffic & $\begin{array}{l}\text { Distance to the nearest road }(0.05-15 \\
\mathrm{km})\end{array}$ & Any available road \\
\hline Population & $\operatorname{Sum}(0.5-3 \mathrm{~km})$ & Population in block groups \\
\hline Land use (Urban) & Percent $(0.05-15 \mathrm{~km})$ & Urban or built-up land, etc. \\
\hline Land use (Rural) & Percent $(0.05-15 \mathrm{~km})$ & Agriculture, forest, water, etc. \\
\hline Source & Distance to the nearest source & Coastline, railroad, airport, etc. \\
\hline Emission & $\begin{array}{l}\text { Sum of cite-specific facility emissions } \\
(3-30 \mathrm{~km})\end{array}$ & $\mathrm{PM}_{2.5}, \mathrm{PM}_{10}, \mathrm{CO}, \mathrm{SO}_{2}, \mathrm{NO}_{\mathrm{x}}$ \\
\hline Vegetation & Quantiles $(0.5-10 \mathrm{~km})$ & Normalized Difference Vegetation Index \\
\hline Imperviousness & Percent $(0.05-5 \mathrm{~km})$ & Impervious surface value \\
\hline Elevation & $\begin{array}{l}\text { Counts of points above/below a } \\
\text { threshold }(1-5 \mathrm{~km})\end{array}$ & Elevation value \\
\hline
\end{tabular}

${ }^{a}$ For detailed description of the geographic variables, please go to Kim et al. (2020). 
Table S2. Candidate predictor variables of satellite estimates

\begin{tabular}{|c|c|c|c|c|c|}
\hline Pollutant & Years & Resolution & Instrument & Level & Source \\
\hline $\mathrm{PM}_{2.5}$ & 2014 & $0.1^{\circ}$ & $\begin{array}{l}\text { Multiple } \\
\text { instruments }\end{array}$ & Surface & $\begin{array}{l}\underline{\text { http://fizz.phys.dal.ca/ atmos/martin/?pag }} \\
\underline{\text { e id }=140}\end{array}$ \\
\hline $\mathrm{NO}_{2}{ }^{\mathrm{a}}$ & 2015 & $0.1^{\circ}$ & $\mathrm{OMI}^{\mathrm{c}}$ & Column & http://www.temis.nl/airpollution/no2.html \\
\hline $\mathrm{SO}_{2}$ & 2015 & $0.25^{\circ}$ & OMI & Column & $\begin{array}{l}\text { https://disc.gsfc.nasa.gov/datacollection/O } \\
\text { MSO2_CPR_003.html }\end{array}$ \\
\hline $\mathrm{HCHO}^{\mathrm{b}}$ & $\begin{array}{l}2005- \\
2016\end{array}$ & $0.1^{\circ}$ & OMI & Column & $\begin{array}{l}\text { https://disc.gsfc.nasa.gov/datasets/OMHC } \\
\underline{\text { HO_V003/summary }}\end{array}$ \\
\hline $\mathrm{CO}$ & 2015 & $0.25^{\circ}$ & MOPITT $^{d}$ & Surface & https://eosweb.larc.nasa.gov/datapool \\
\hline
\end{tabular}

aBoth 1-year and 3-year averages calculated;

bLong term (12-year) average only;

'Ozone Monitoring Instrument;

${ }^{\mathrm{d}}$ Measurements of Pollution in the Troposphere.

For detailed description of the satellite data, please go to Kim et al. (2020). 
Table S3. Categories of point of interest $(\mathrm{POI})$ data $(\mathrm{n}=90)$ used in LUR models

\begin{tabular}{|c|c|c|}
\hline \multicolumn{3}{|c|}{90 Google POI categories (buffers: $100,250,500,750,1000 \mathrm{~m}$ ) } \\
\hline accounting & electrician & night club \\
\hline airport & electronics_store & painter \\
\hline amusement_park & embassy & park \\
\hline aquarium & fire_station & parking \\
\hline art_gallery & florist & pet_store \\
\hline atm & funeral home & pharmacy \\
\hline bakery & furniture_store & physiotherapist \\
\hline bank & gas_station & plumber \\
\hline bar & gym & police \\
\hline beauty_salon & hair_care & post_office \\
\hline bicycle_store & hardware_store & real_estate_agency \\
\hline book_store & hindu_temple & restaurant \\
\hline bowling_alley & home_goods_store & roofing_contractor \\
\hline bus_station & hospital & rv_park \\
\hline cafe & insurance_agency & school \\
\hline campground & jewelry_store & shoe_store \\
\hline car_dealer & laundry & shopping_mall \\
\hline car_rental & lawyer & spa \\
\hline car_repair & library & stadium \\
\hline car_wash & liquor_store & storage \\
\hline casino & local_government_office & store \\
\hline cemetery & locksmith & subway_station \\
\hline church & lodging & supermarket \\
\hline city_hall & meal_delivery & synagogue \\
\hline clothing_store & meal_takeaway & taxi_stand \\
\hline convenience_store & mosque & train station \\
\hline courthouse & movie_rental & transit_station \\
\hline dentist & movie theater & travel_agency \\
\hline department_store & moving_company & veterinary_care \\
\hline doctor & museum & zoo \\
\hline
\end{tabular}


Table S4. Categories of Google street view imagery (GSV) data used in LUR models $(\mathrm{n}=57)$

\begin{tabular}{|l|l|l|}
\hline \multicolumn{2}{|c|}{57 outdoor Google Street View (GSV) categories (percentage of features per image) } \\
\hline wall & railing & airplane \\
\hline building & box & dirt.track \\
\hline sky & signboard & pole \\
\hline tree & sand & land \\
\hline road & skyscraper & van \\
\hline windowpane & path & ship \\
\hline grass & runway & fountain \\
\hline sidewalk & river & canopy \\
\hline person & bridge & swimming.pool \\
\hline earth & flower & waterfall \\
\hline mountain & hill & tent \\
\hline plant & palm & minibike \\
\hline car & boat & food \\
\hline water & hovel & pot \\
\hline house & bus & animal \\
\hline sea & truck & bicycle \\
\hline field & tower & lake \\
\hline fence & awning & sculpture \\
\hline rock & streetlight & traffic.light \\
\hline & & \\
\hline
\end{tabular}


Table S5. Machine learning hyperparameters

\begin{tabular}{|l|l|l|l|}
\hline Model & Hyperparameters & Definition & Tested Range \\
\hline \multirow{4}{*}{$\begin{array}{l}\text { Gradient } \\
\text { Boosting }\end{array}$} & n_estimators & The number of boosting stages & range $(10,460,10)$ \\
\cline { 2 - 4 } & max_features & $\begin{array}{l}\text { The number of features to consider while } \\
\text { searching for a best split }\end{array}$ & range $(10,180,10)$ \\
\cline { 2 - 4 } & max_depth & $\begin{array}{l}\text { The maximum depth of a tree to control over- } \\
\text { fitting }\end{array}$ & range $(1,10)$ \\
\cline { 2 - 4 } & min_samples_split & $\begin{array}{l}\text { The minimum number of samples required to split } \\
\text { an internal node }\end{array}$ & range $(2,30)$ \\
\cline { 2 - 4 } & min_samples_leaf & $\begin{array}{l}\text { The minimum number of samples required to be } \\
\text { at a leaf node }\end{array}$ & range $(1,30)$ \\
\hline
\end{tabular}


Table S6. Descriptive statistics of the concentrations for all criteria pollutants in the US EPA monitors.

\begin{tabular}{|l|c|c|c|c|c|c|}
\hline Descriptive statistics & $\mathbf{N O}_{\mathbf{2}}(\mathbf{p p b})$ & $\mathbf{P M}_{\mathbf{2 . 5}}\left(\mathbf{\mu g} / \mathbf{m}^{\mathbf{3}}\right)$ & $\mathbf{O}_{\mathbf{3}}(\mathbf{p p b})$ & $\mathbf{C O}(\mathbf{p p m})$ & $\mathbf{P M}_{\mathbf{1 0}}\left(\mathbf{\mu g} / \mathbf{m}^{\mathbf{3}}\right)$ & $\mathbf{S O}_{\mathbf{2}}(\mathbf{p p b})$ \\
\hline Number of monitors & 320 & 757 & 821 & 196 & 456 & 367 \\
\hline Mean concentrations & 7.8 & 7.7 & 44.2 & 0.3 & 16 & 1.2 \\
\hline Standard deviation & 4.9 & 2.4 & 5.2 & 0.1 & 7.9 & 0.9 \\
\hline Min & 0.4 & 1.8 & 25.4 & 0.1 & 3.2 & 0.1 \\
\hline 25th percentile & 3.9 & 6.2 & 41.1 & 0.3 & 10.9 & 0.7 \\
\hline Median & 7.1 & 8.0 & 44.5 & 0.3 & 15.6 & 1.0 \\
\hline 75th percentile & 10.9 & 9.2 & 47.5 & 0.3 & 19.7 & 1.4 \\
\hline Max & 22.3 & 18.0 & 57.3 & 0.7 & 46.9 & 6.4 \\
\hline
\end{tabular}


Table S7. The top 30 variables selected as predictor variables for PLS-K models (an example of the traditional scenario).

\begin{tabular}{|c|c|c|c|c|c|}
\hline $\mathrm{NO}_{2}$ & $\mathrm{PM}_{2.5}$ & $\mathrm{O}_{3}$ & $\mathrm{CO}$ & $\mathrm{PM}_{10}$ & $\mathrm{SO}_{2}$ \\
\hline imp_a03000 & elev_elevation & elev_elevation & imp_a01000 & imp_a00750 & em_PM25_s 15000 \\
\hline rlu_dev_med_p05000 & $\log 10 \_m$ to_rr & lu_shrub_p15000 & imp_a00750 & rlu_evergreen_p05000 & em_PM10_s03000 \\
\hline lu_oth_urban_p15000 & $\log 10$ m to a 3 a 3 & sathcho_2005_2016 & imp_a00500 & $\log 10 \_\mathrm{m}$ to $\mathrm{rr}$ & rlu_decid_forest_p05000 \\
\hline rlu_dev hi p03000 & sathcho_2005_2016 & ndvi_q50_a07500 & $\log 10 \mathrm{~m}$ to 1 airp & imp_a00400 & $\log 10 \mathrm{~m}$ to $\mathrm{rr}$ \\
\hline $\log 10 \mathrm{~m}$ to $\mathrm{a} 1$ & elev $1 \mathrm{k}$ at elev & lu_shrub_p03000 & satno2_2015 & imp_a00300 & satpm25_2014 \\
\hline pop_s 15000 & imp_a00300 & ndvi_winter_a10000 & rlu_crop_p03000 & imp_a00150 & rlu_evergreen_p05000 \\
\hline rlu_dev_hi_p01000 & satno2_2015 & satno2_2015 & imp_a00150 & rlu_dev_med_p05000 & lu_industrial_p05000 \\
\hline $\log 10 \_\mathrm{m}$ to_rr & rlu_shrub_p05000 & satco_2015 & $11 \_$a23_s05000 & lu_crop_p03000 & lu_shrub_p05000 \\
\hline satco_2015 & rlu_evergreen_p01000 & lu_crop_p15000 & rlu_dev_med_p03000 & elev_elevation & lu_comm_p10000 \\
\hline rlu_dev_med_p00050 & rlu_dev_med_p03000 & $\log 10$ m to 1 airp & lu_herb_range p 03000 & satno2_2015 & elev_1k_at_elev \\
\hline elev_elevation & rlu_dev_med_p05000 & rlu_dev_hi_p03000 & ndvi_q75_a00250 & rlu_dev_lo_p00300 & lu_oth_urban_p01500 \\
\hline rlu_grass_p05000 & lu_industrial_p15000 & em_SO2_s30000 & ndvi_q75_a00500 & lu_crop_p15000 & lu_comm_p05000 \\
\hline lu_shrub_p00750 & $11 \_$a23_s01500 & lu_industrial_p15000 & rlu_pasture p00500 & lu_oth_urban_p10000 & lu_bays_p10000 \\
\hline ndvi_q50_a00250 & $\log 10 \mathrm{~m}$ to $\mathrm{comm}$ & lu_mix forest p01500 & lu_resi_p05000 & pop s05000 & rlu_dev_med_p00050 \\
\hline rlu_dev lo_p00100 & lu_oth_urban_p05000 & rlu_dev_med_p01000 & pop_s10000 & lu_oth_urban_p15000 & rlu_dev_open_p00050 \\
\hline lu_bays_p10000 & rlu_dev_lo_p00300 & rlu_evergreen_p00150 & lu_transport_p10000 & lu_green_p00300 & tl_s15000 \\
\hline lu_green_p15000 & lu_resi_p00300 & $\log 10$ m to a 1 a 1 & rlu_evergreen_p05000 & lu_oth_urban_p03000 & pop_s15000 \\
\hline rlu_pasture_p00750 & lu_shrub_p01500 & lu_comm_p15000 & $\log 10 \_m$ to $a 3$ & $\mathrm{tl} \_\mathrm{s} 05000$ & rlu_dev_lo_p00300 \\
\hline elev_5k_above & lu_crop_p15000 & lu_mix_range_p10000 & ndvi_summer_a10000 & ndvi_summer_a00500 & rlu_water_p00500 \\
\hline ndvi_q50_a02500 & em_SO2_s30000 & rlu_crop_p00300 & lu_crop_p00150 & rlu_dev_open_p03000 & lu_crop_p15000 \\
\hline lu_mix range p05000 & lu_crop p10000 & satso2_2015 & lu_resi_p00050 & rlu pasture p05000 & $\log 10$ m to a 3 a3 \\
\hline sathcho_2005_2016 & elev_5k_above & rlu_dev_open_p05000 & $\log 10$ m to $\mathrm{a} 1$ & rlu_shrub_p05000 & lu_resi_p15000 \\
\hline lu_unspec_p15000 & $\log 10 \mathrm{~m}$ to $\mathrm{a} 2$ & rlu_dev hi_p00050 & lu_mix forest p01500 & satco_2015 & lu_green_p01500 \\
\hline lu_herb_range p 15000 & lu_crop_p01500 & rlu_decid_forest_p00100 & intersect_a2_a3 s01000 & intersect_a2_a2_s03000 & sathcho_2005_2016 \\
\hline rlu_woody_wetland_p05000 & rlu_mix_forest_p05000 & lu_resi_p01500 & lu_stream_p15000 & ndvi_q50_a10000 & $\log 10$ m to a1 a3 \\
\hline tl_s00300 & lu_stream_p15000 & lu_comm_p00100 & lu_stream_p10000 & lu_transition p15000 & lu_stream_p00750 \\
\hline ndvi_q25_a01000 & rlu pasture p03000 & intersect_a1_a2_s03000 & lu transition p 01500 & lu_stream_p10000 & elev_elevation \\
\hline ndvi_q50_a07500 & lu_mix_forest_p05000 & rlu_grass_p05000 & lu_bays_p15000 & elev_5k_above & satno2_2015 \\
\hline lu_grove $p 15000$ & tl_s00400 & rlu_grass_p00400 & sathcho_2005_2016 & sathcho_2005_2016 & rlu_pasture_p00100 \\
\hline rlu_water_p00300 & rlu_woody_wetland_p05000 & lu_industrial_p01000 & lu_oth_urban_p01500 & lu_mix forest p 10000 & lu_transport_p00300 \\
\hline
\end{tabular}



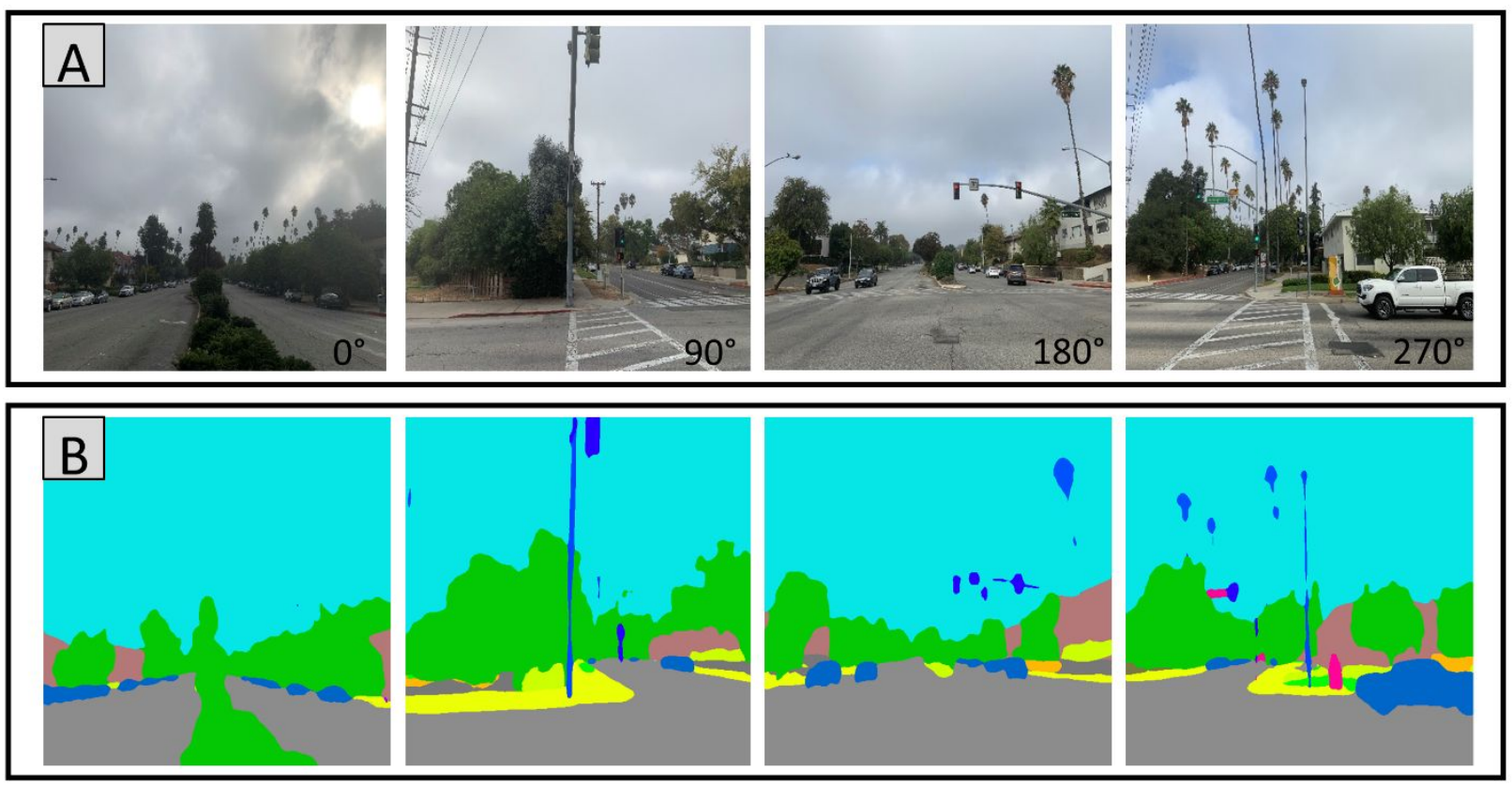

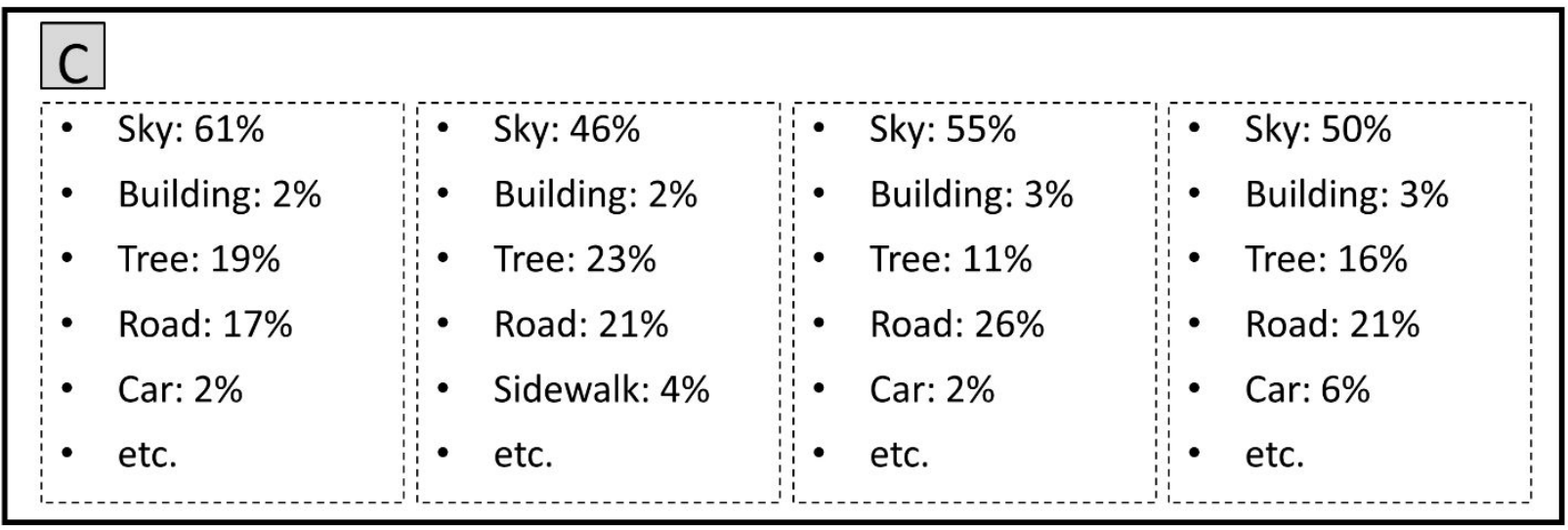

Figure S1. Example 1 (urban street) of extracted street view features (photos taken by the authors) using Pyramid Scene Parsing Network (PSPNet) algorithm; this example was used to demonstrate the process of extracted Google Street View (GSV) features. (A) Retrieved GSV panorama from 4 heading directions $\left(0^{\circ}, 90^{\circ}, 180^{\circ}, 270^{\circ}\right)$ per location of interest. (B) Corresponding extracted features using PSPNet scene parsing. (C) Major estimated feature percentages of each image based on the GSV classes. 

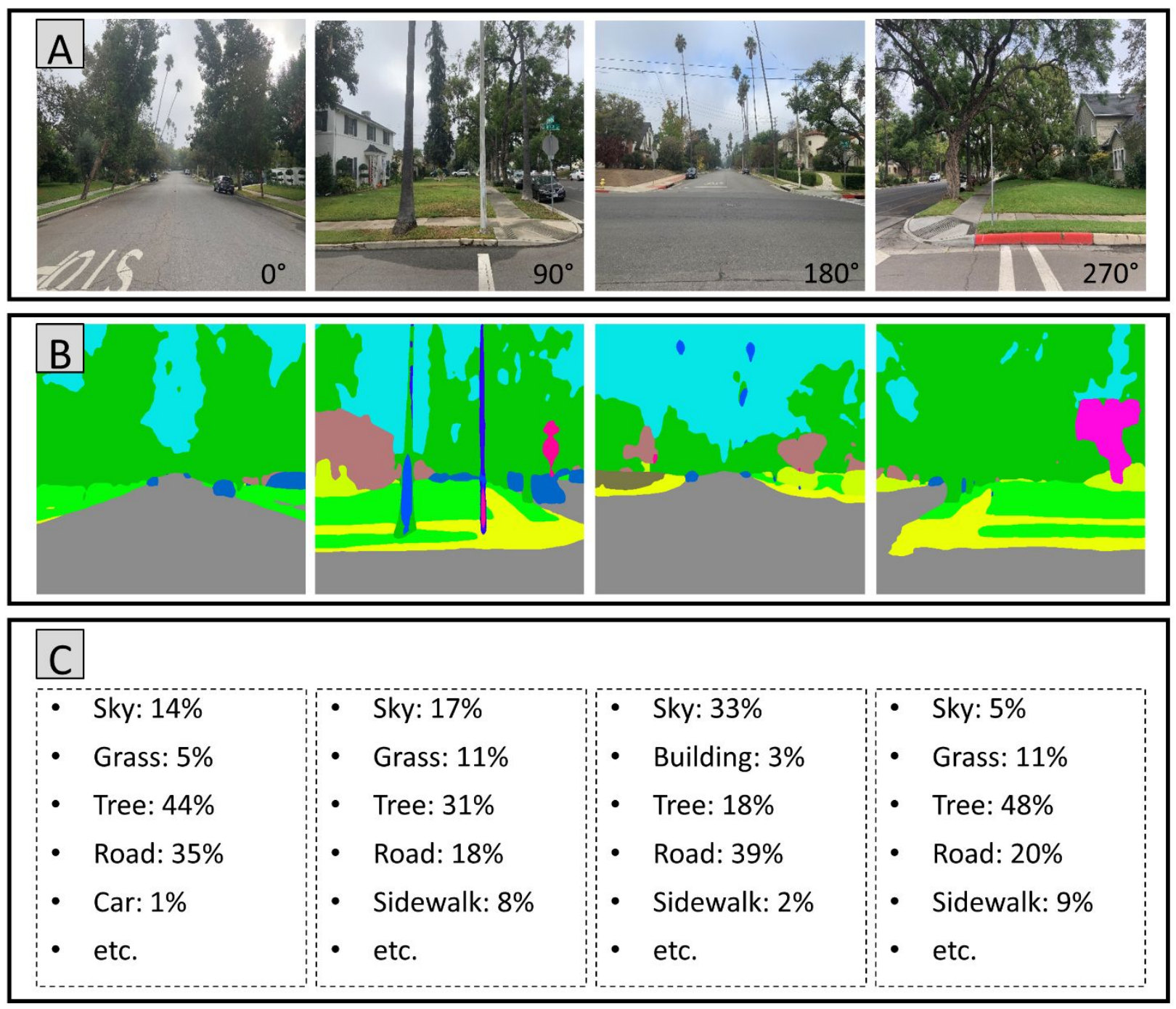

Figure S2. Example 2 (residential environment) of extracted street view features (photos taken by the authors) using Pyramid Scene Parsing Network (PSPNet) algorithm; this example was used to demonstrate the process of extracted Google Street View (GSV) features. (A) Retrieved GSV panorama from 4 heading directions $\left(0^{\circ}, 90^{\circ}, 180^{\circ}, 270^{\circ}\right)$ per location of interest. (B) Corresponding extracted features using PSPNet scene parsing. (C) Major estimated feature percentages of each image based on the GSV classes. 


\section{Built types}

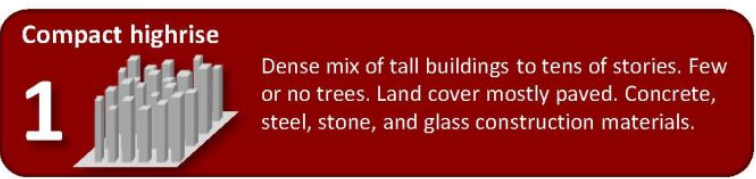

\section{Compact midrise}

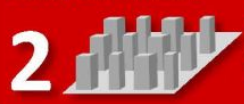

ense mix of midrise buildings (3-9 stories). Few

or no trees. Land cover mostly paved. Stone,

brick, tile, and concrete construction materials.

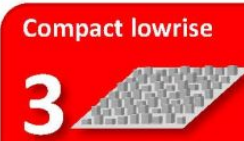

Dense mix of lowrise buildings (1-3 stories). Few or no trees. Land cover mostly paved. Stone. brick, tile, and concrete construction materials.
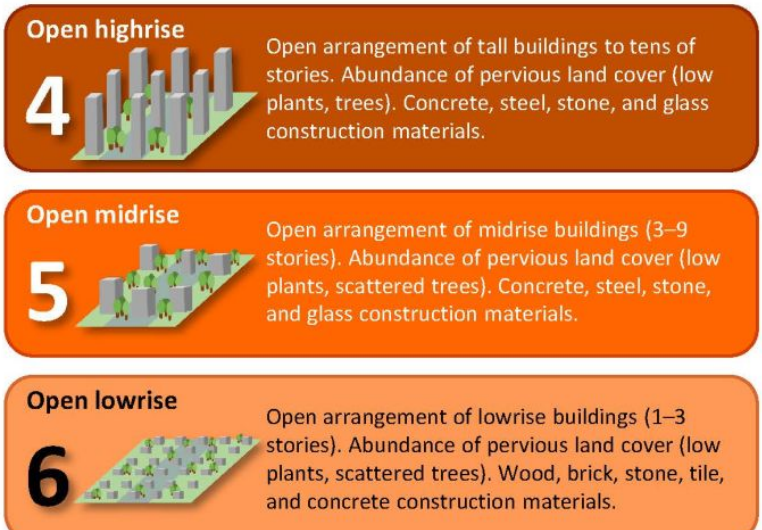

Open arrangement of lowrise buildings (1-3 stories). Abundance of pervious land cover (low plants, scattered trees). Wood, brick, stone, tile, and concrete construction materials.
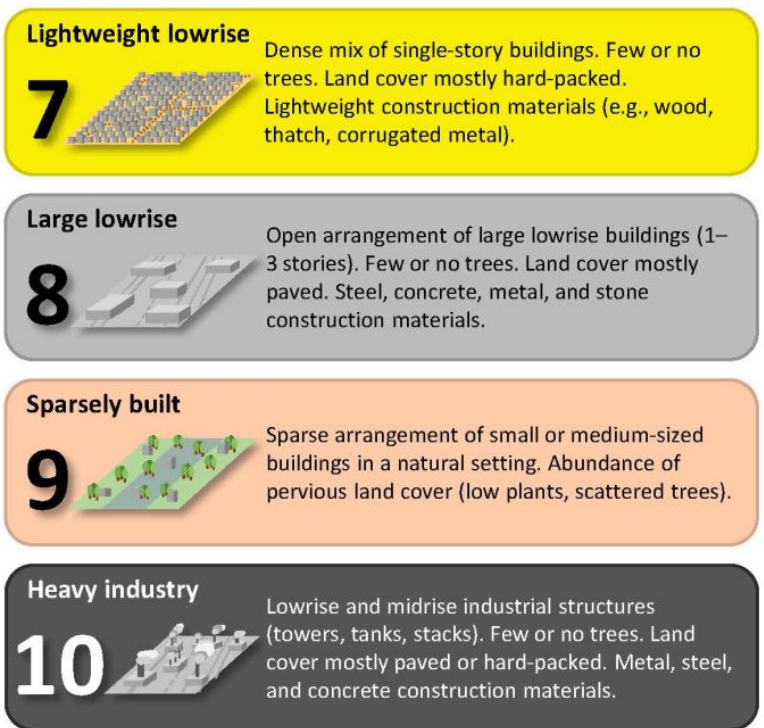

\section{Land cover types}
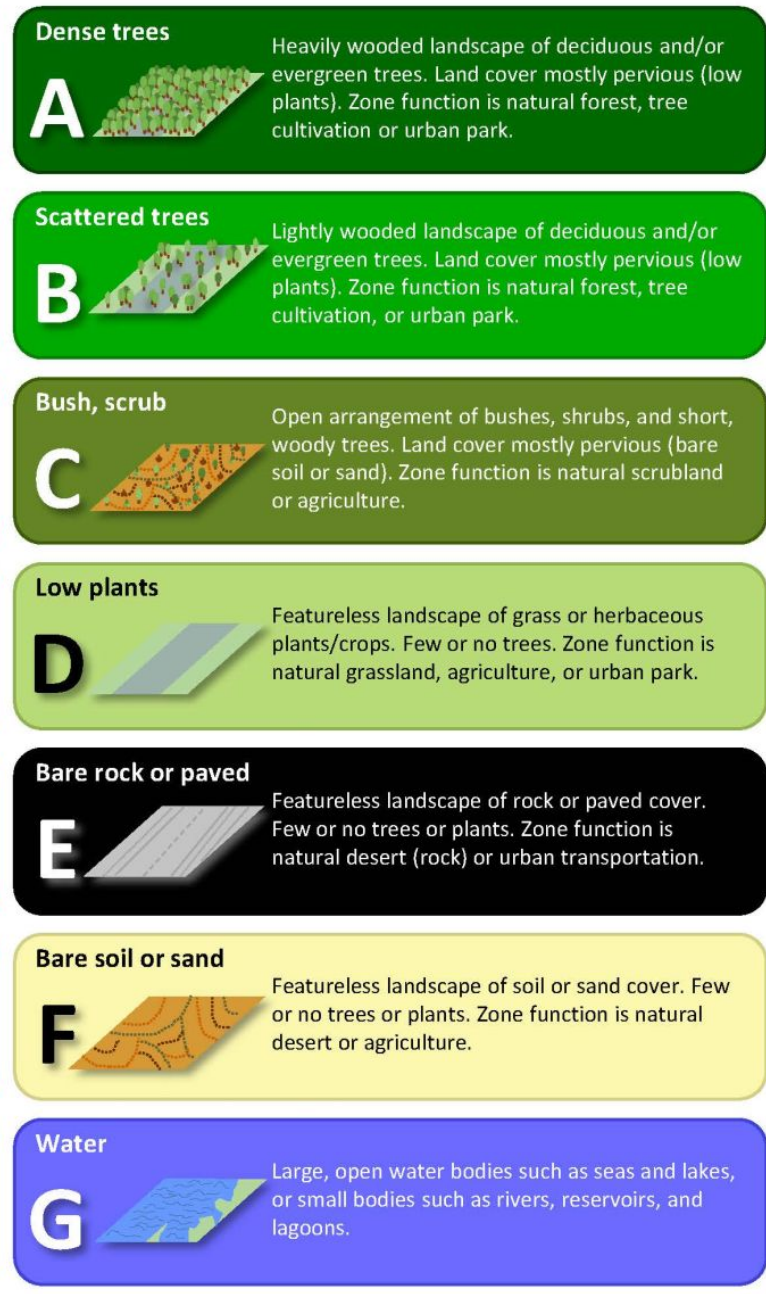

\section{VARIABLE LAND COVER PROPERTIES}

Variable or ephemeral land cover properties that change significantly with synoptic weather patterns, agricultural practices, and/or seasonal cycles.

\section{b. bare trees Leafless deciduous trees (e.g., winter). Increased sky view factor. Reduced albedo.}

s. snow cover

Snow cover $>10 \mathrm{~cm}$ in depth. Low admittance. High albedo.

d. dry ground Parched soil. Low admittance. Large Bowen ratio. Increased albedo.

w. wet ground ratio. Reduced albedo.

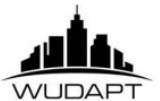

Figure S3. Local climate zone (LCZ) classification (Demuzere et al., 2020). 


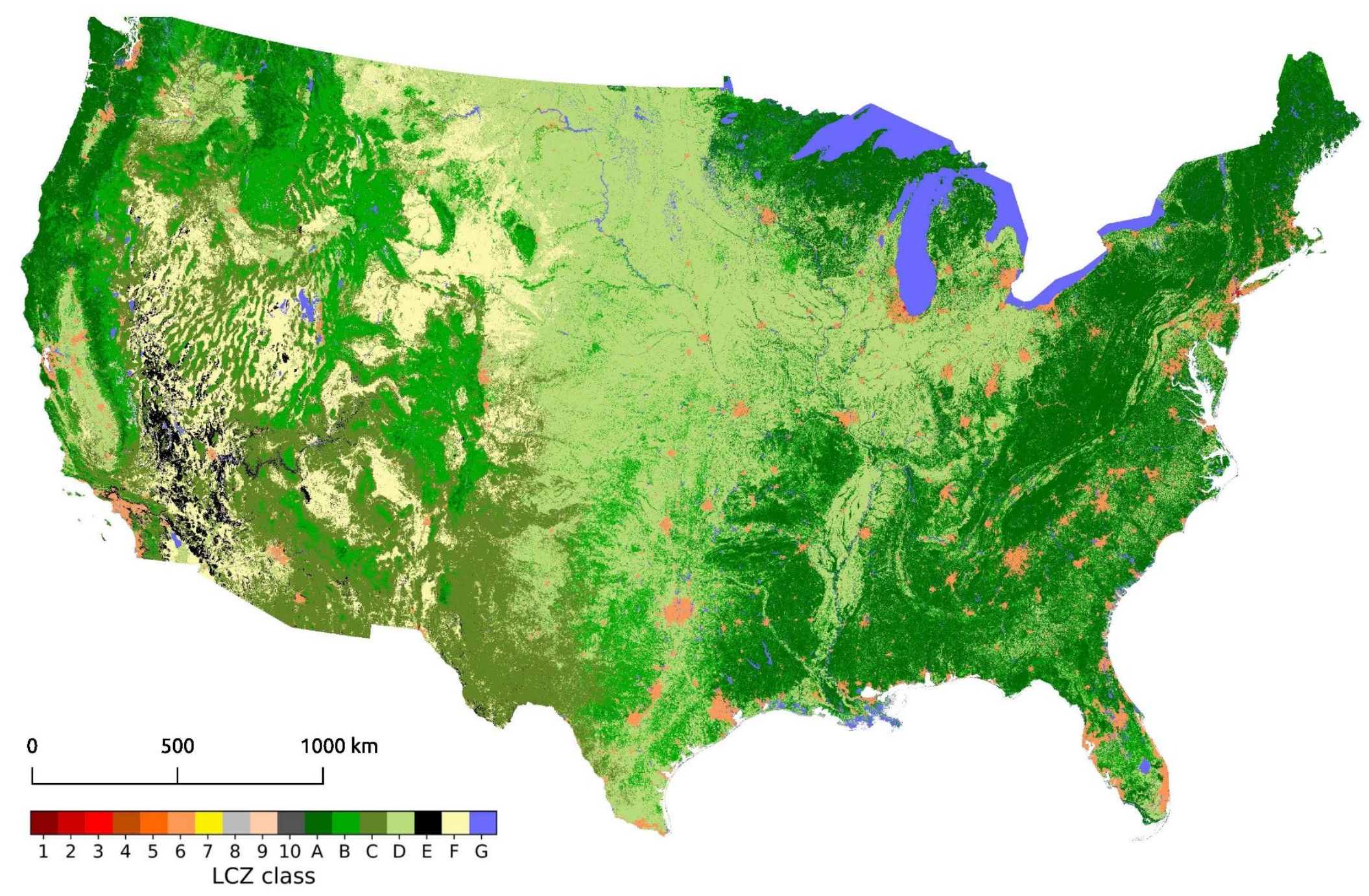

Figure S4. Local climate zone (LCZ) surface for the continental US (Demuzere et al., 2020). 

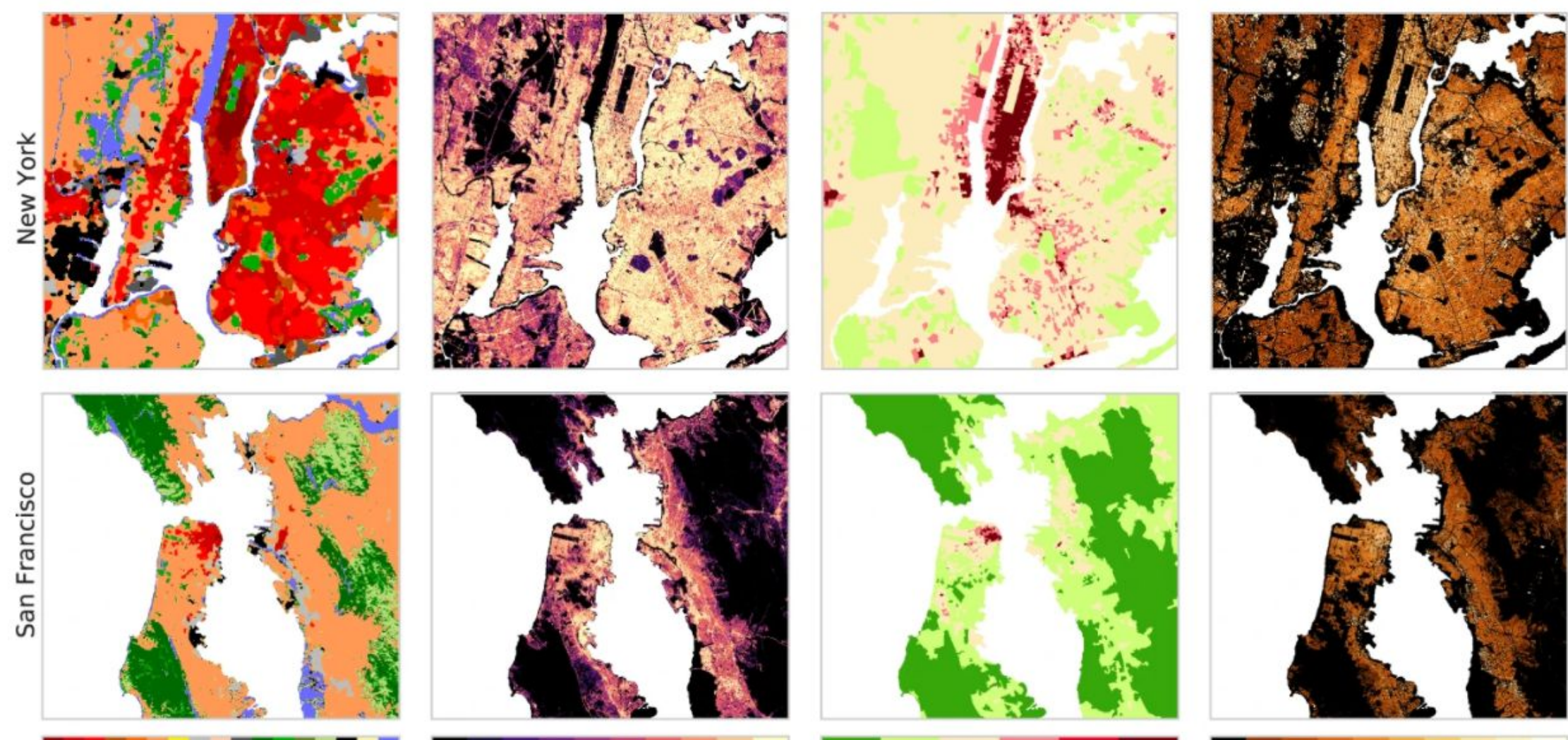

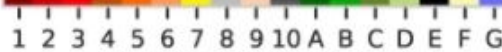
$\mathrm{LCZ}$
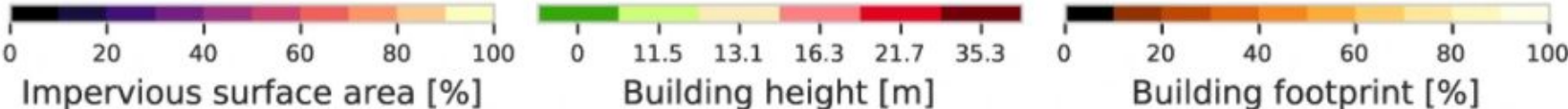

Figure S5. LCZ surface for two selected urban areas in the US: New York (top) and San Francisco (bottom). The impervious surface area, building height, and building footprint are shown as references (Demuzere et al., 2020). 


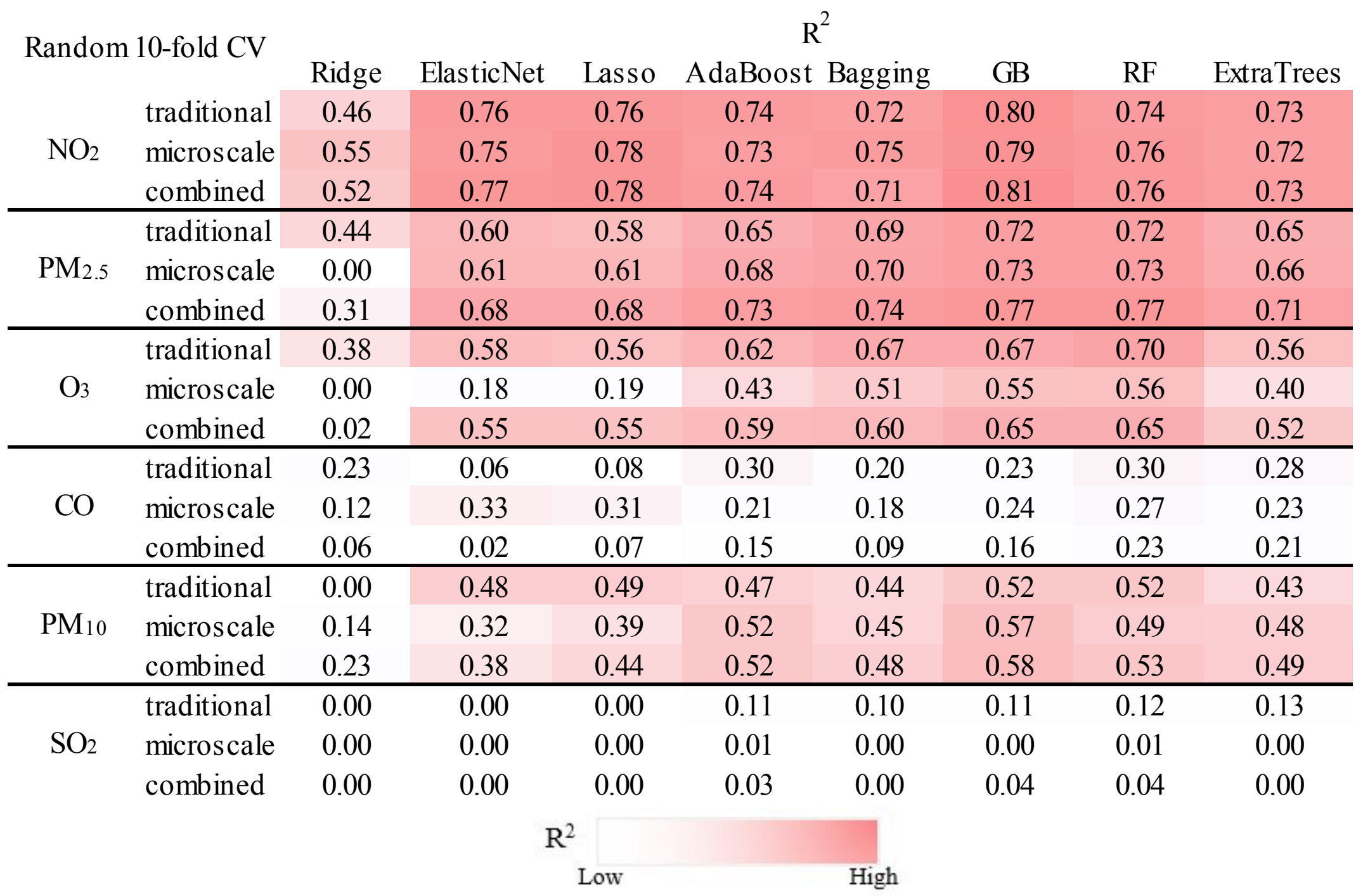

Figure S6. Random 10-fold CV results $\left(\mathrm{R}^{2}\right)$ of criteria pollutants of the eight ML algorithms. GB stands for Gradient Boosting while RF stands for Random Forest. 


\section{RMSE}

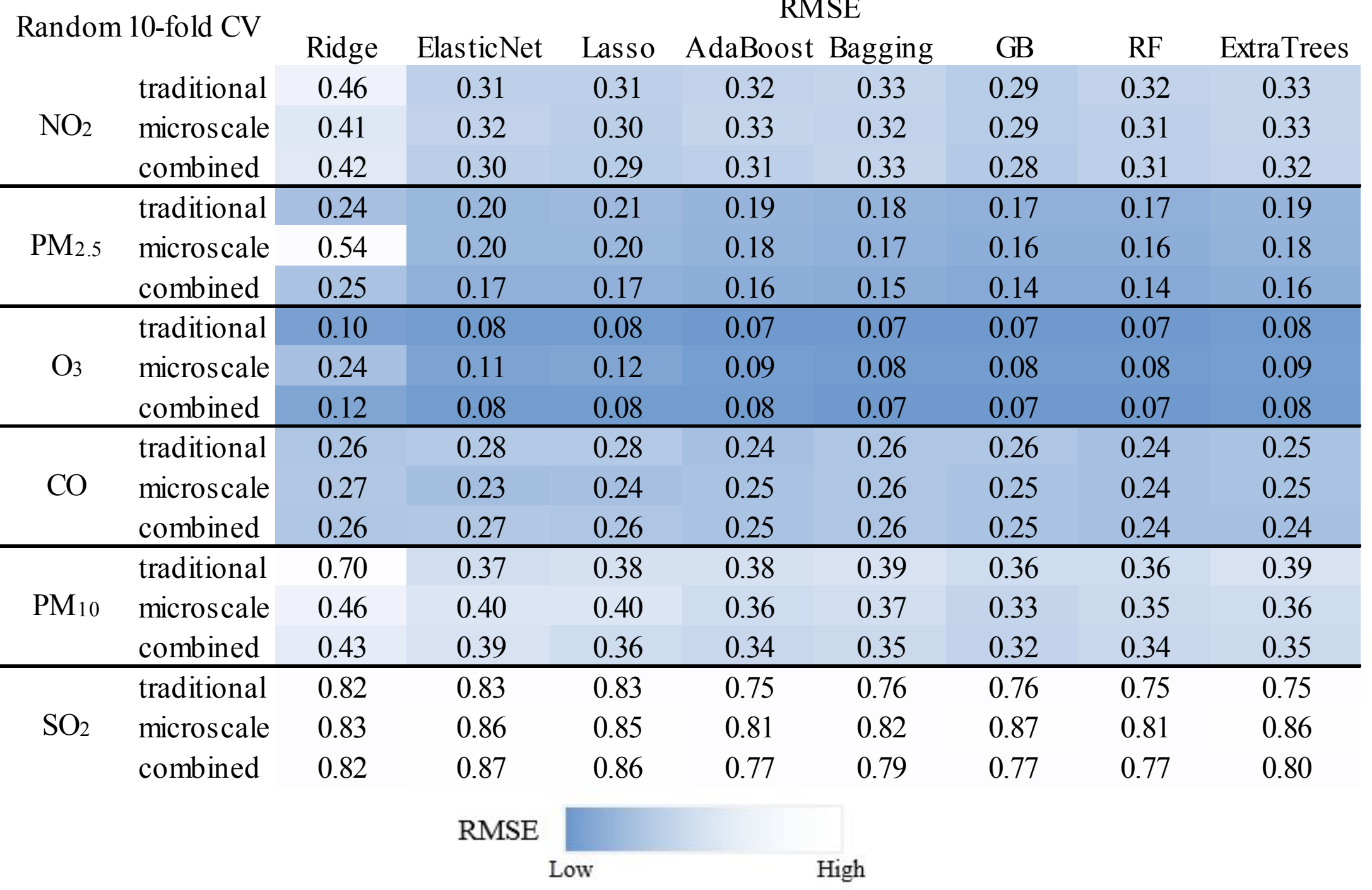

Figure S7. Random 10-fold CV results (RMSE) of criteria pollutants of the eight ML algorithms. GB stands for Gradient Boosting while RF stands for Random Forest. 


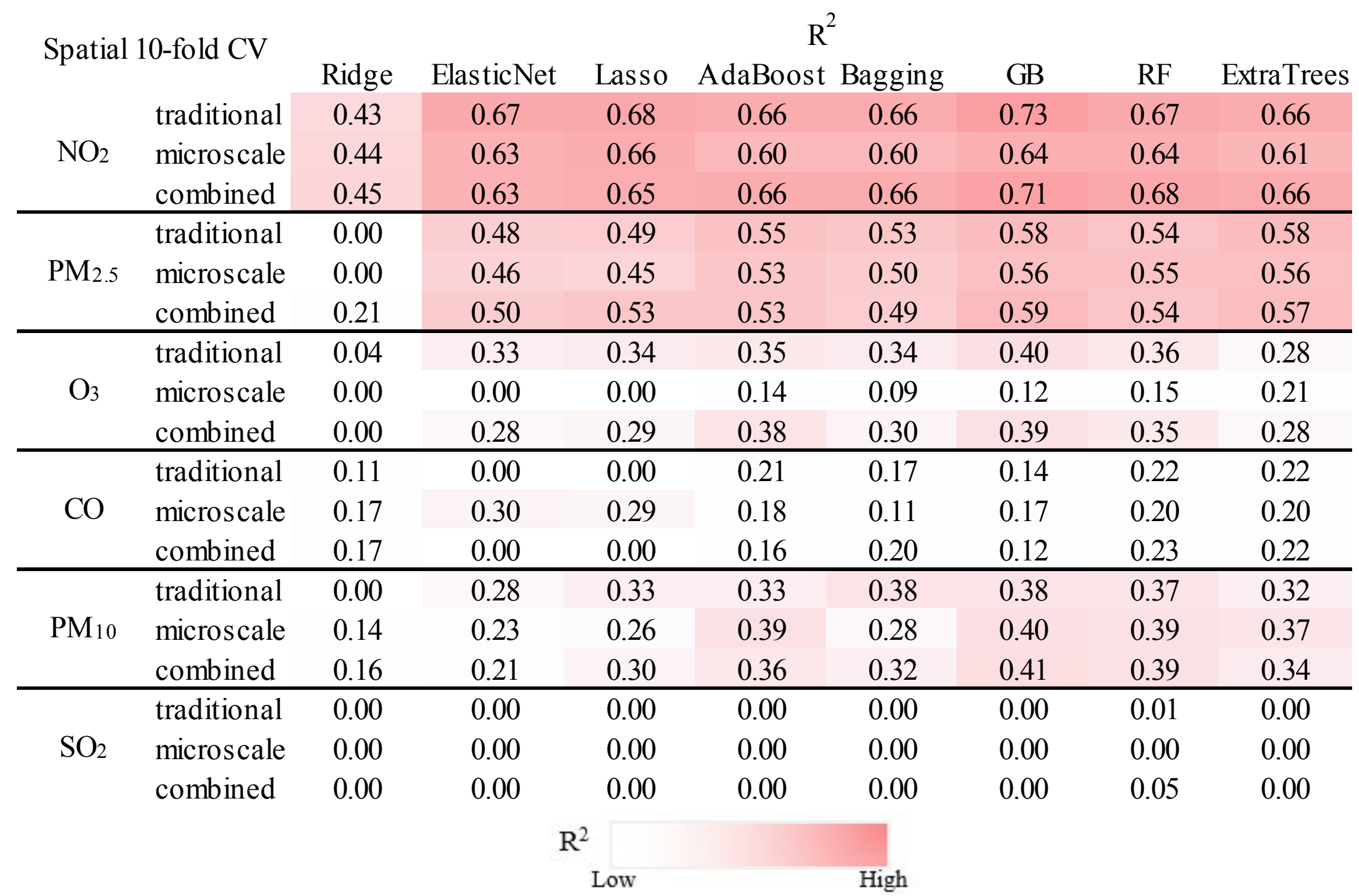

Figure S8. Spatial 10-fold CV results $\left(\mathrm{R}^{2}\right)$ of criteria pollutants of the eight $\mathrm{ML}$ algorithms. GB stands for Gradient Boosting while $\mathrm{RF}$ stands for Random Forest. 


\begin{tabular}{|c|c|c|c|c|c|c|c|c|c|}
\hline \multirow{2}{*}{\multicolumn{2}{|c|}{ Spatial 10-fold CV }} & \multicolumn{8}{|c|}{ RMSE } \\
\hline & & Ridge & Elas ticNet & Lasso & AdaBoost & Bagging & GB & $\mathrm{RF}$ & ExtraTrees \\
\hline \multirow{3}{*}{$\mathrm{NO}_{2}$} & traditional & 0.48 & 0.36 & 0.36 & 0.37 & 0.37 & 0.33 & 0.36 & 0.37 \\
\hline & microscale & 0.47 & 0.39 & 0.37 & 0.40 & 0.40 & 0.38 & 0.38 & 0.40 \\
\hline & combined & 0.47 & 0.38 & 0.37 & 0.37 & 0.37 & 0.35 & 0.35 & 0.37 \\
\hline \multirow{3}{*}{$\mathrm{PM}_{2.5}$} & traditional & 0.31 & 0.23 & 0.23 & 0.21 & 0.22 & 0.20 & 0.21 & 0.20 \\
\hline & microscale & 0.82 & 0.23 & 0.23 & 0.22 & 0.22 & 0.21 & 0.21 & 0.21 \\
\hline & combined & 0.28 & 0.22 & 0.22 & 0.22 & 0.23 & 0.20 & 0.21 & 0.21 \\
\hline \multirow{3}{*}{$\mathrm{O}_{3}$} & traditional & 0.12 & 0.10 & 0.10 & 0.10 & 0.10 & 0.09 & 0.09 & 0.10 \\
\hline & microscale & 0.44 & 0.13 & 0.14 & 0.11 & 0.11 & 0.11 & 0.11 & 0.11 \\
\hline & combined & 0.13 & 0.10 & 0.10 & 0.09 & 0.10 & 0.09 & 0.10 & 0.10 \\
\hline \multirow{3}{*}{$\mathrm{CO}$} & traditional & 0.28 & 0.37 & 0.36 & 0.27 & 0.28 & 0.28 & 0.27 & 0.27 \\
\hline & microscale & 0.28 & 0.25 & 0.25 & 0.27 & 0.28 & 0.28 & 0.27 & 0.27 \\
\hline & combined & 0.28 & 0.31 & 0.32 & 0.28 & 0.27 & 0.28 & 0.26 & 0.27 \\
\hline \multirow{3}{*}{$\mathrm{PM}_{10}$} & traditional & 0.84 & 0.42 & 0.41 & 0.41 & 0.39 & 0.39 & 0.39 & 0.41 \\
\hline & microscale & 0.46 & 0.44 & 0.43 & 0.39 & 0.42 & 0.38 & 0.39 & 0.39 \\
\hline & combined & 0.45 & 0.44 & 0.41 & 0.40 & 0.41 & 0.38 & 0.39 & 0.40 \\
\hline \multirow{5}{*}{$\mathrm{SO}_{2}$} & traditional & 0.78 & 0.94 & 0.92 & 0.74 & 0.77 & 0.77 & 0.73 & 0.74 \\
\hline & microscale & 0.84 & 0.87 & 0.85 & 0.76 & 0.83 & 0.80 & 0.78 & 0.83 \\
\hline & combined & 0.83 & 0.90 & 0.88 & 0.77 & 0.78 & 0.74 & 0.72 & 0.77 \\
\hline & & & RMSE & & & & & & \\
\hline & & & \multicolumn{2}{|c|}{ Low } & \multicolumn{2}{|c|}{ High } & & & \\
\hline
\end{tabular}

Figure S9. Spatial 10-fold CV results (RMSE) of criteria pollutants of the eight ML algorithms. GB stands for Gradient Boosting while RF stands for Random Forest. 


\begin{tabular}{cccccccc}
\multicolumn{2}{c}{ Random vs Spatial } & \multicolumn{4}{c}{$\mathrm{R}^{2}$} & \multicolumn{4}{c}{ RMSE } \\
& & \multicolumn{1}{c}{ SW-K } & PLS-K & ML-K & SW-K & PLS-K & ML-K \\
& traditional & 0.05 & 0.13 & 0.11 & -0.05 & -0.10 & -0.09 \\
$\mathrm{NO}_{2}$ & microscale & 0.12 & 0.11 & 0.12 & -0.09 & -0.08 & -0.09 \\
& combined & 0.14 & 0.10 & 0.12 & -0.09 & -0.08 & -0.11 \\
\hline \multirow{3}{*}{$\mathrm{PM}_{2.5}$} & traditional & 0.08 & 0.19 & 0.21 & -0.03 & -0.06 & -0.07 \\
& microscale & 0.08 & 0.26 & 0.18 & -0.02 & -0.07 & -0.07 \\
& combined & 0.08 & 0.15 & 0.23 & -0.03 & -0.04 & -0.08 \\
\hline \multirow{3}{*}{$\mathrm{O}_{3}$} & traditional & 0.71 & 0.36 & 0.27 & -0.06 & -0.03 & -0.03 \\
& microscale & 0.36 & 0.27 & 0.41 & -0.03 & -0.02 & -0.04 \\
& combined & 0.26 & 0.30 & 0.27 & -0.03 & -0.03 & -0.03 \\
\hline \multirow{3}{*}{$\mathrm{CO}$} & traditional & 0.22 & 0.03 & 0.08 & -0.06 & 0.00 & -0.03 \\
& microscale & 0.22 & 0.02 & 0.06 & -0.06 & -0.01 & -0.03 \\
& combined & 0.14 & 0.12 & 0.09 & -0.04 & -0.04 & -0.02 \\
\hline \multirow{3}{*}{$\mathrm{PM}_{10}$} & traditional & 0.15 & 0.18 & 0.25 & -0.05 & -0.07 & 0.13 \\
& microscale & 0.18 & 0.18 & 0.13 & -0.07 & -0.06 & -0.07 \\
& combined & 0.20 & 0.18 & 0.25 & -0.08 & -0.07 & -0.11 \\
\hline \multirow{2}{*}{$\mathrm{SO}_{2}$} & traditional & 0.16 & 0.07 & 0.17 & -0.08 & -0.03 & -0.13 \\
& microscale & 0.16 & 0.03 & 0.37 & -0.08 & -0.01 & -0.18 \\
& combined & 0.07 & 0.02 & 0.02 & -0.03 & -0.01 & -0.06
\end{tabular}

Figure S10. The difference between random and spatial 10-fold CV results of the six criteria pollutants; the value equals random CV minus spatial $\mathrm{CV}$. 


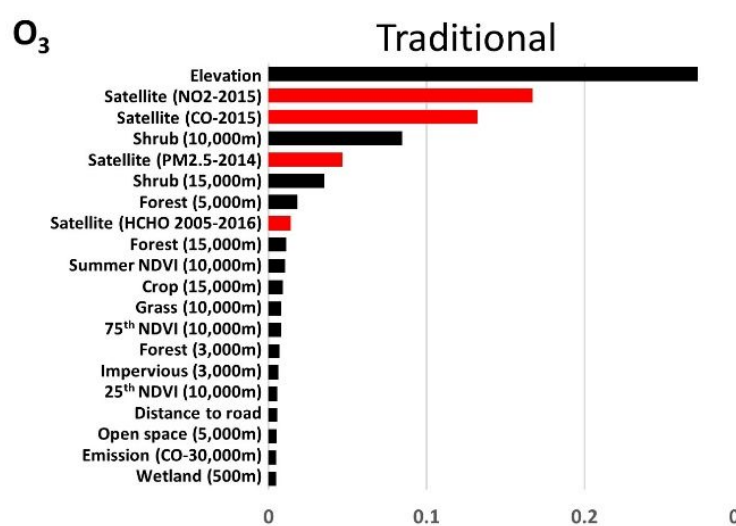

CO

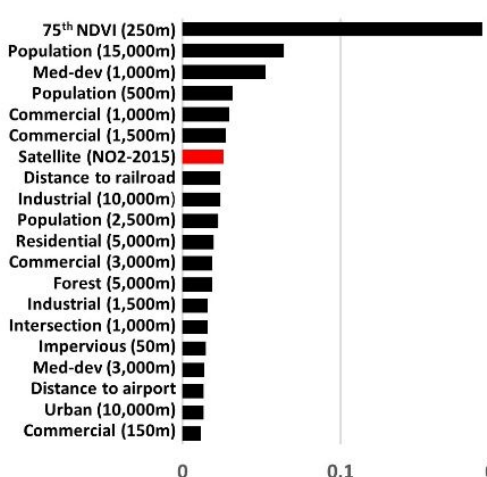

Geographic

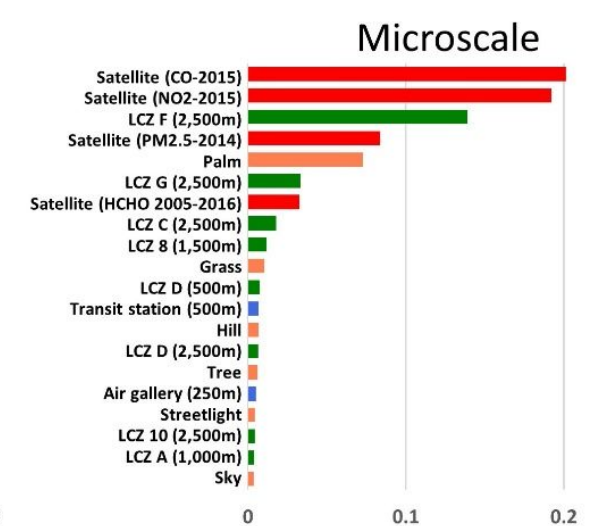

0.2

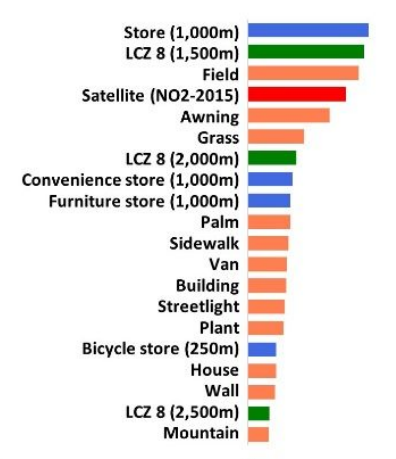

Satellite

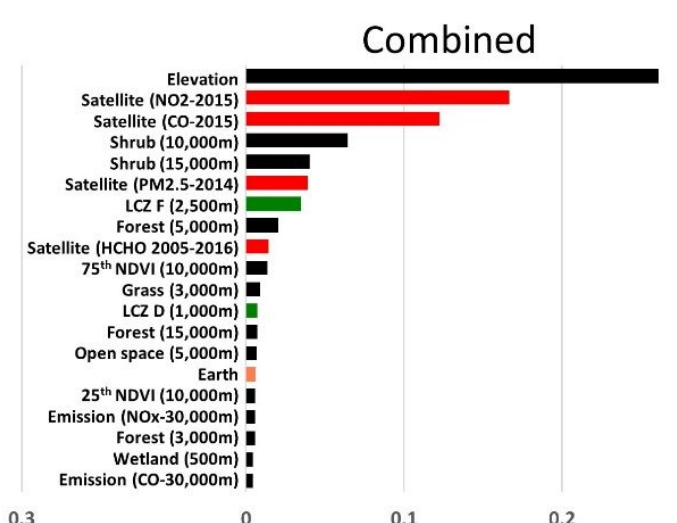

0.3

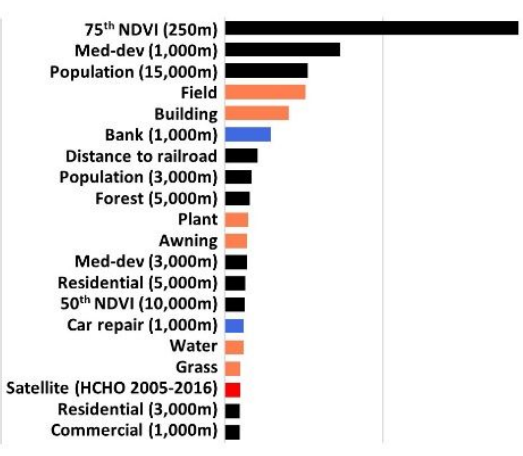

GSV
0.1

0.2

Figure S11. Variable importance of the top 20 variables by variable type (traditional vs. microscale. vs. combined) for the ML-based models of $\mathrm{O}_{3}$ and $\mathrm{CO}$. For example, impervious $(5,000 \mathrm{~m})$ means area of impervious surface within $5,000 \mathrm{~m}$. Satellite $\left(\mathrm{NO}_{2}-2015\right)$ means satellite estimates of $\mathrm{NO}_{2}$ in 2015. LCZ $8(1,500 \mathrm{~m})$ means number of LCZ 8: Large low-rise grids within 1,500m. 


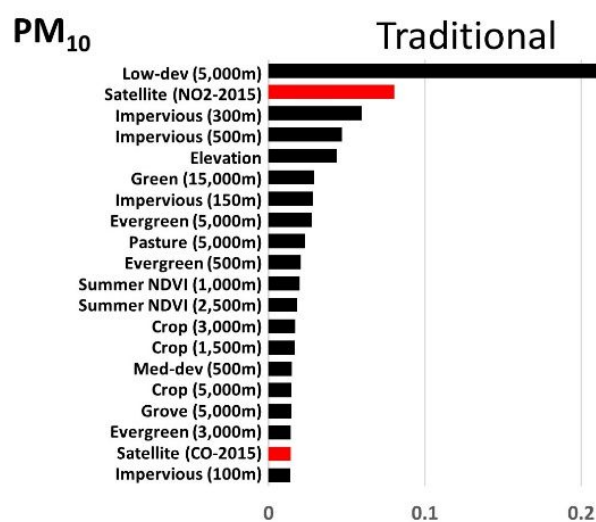

0.2

$\mathrm{SO}_{2}$

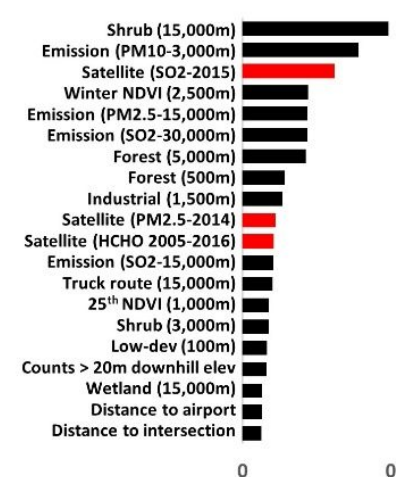

0.1

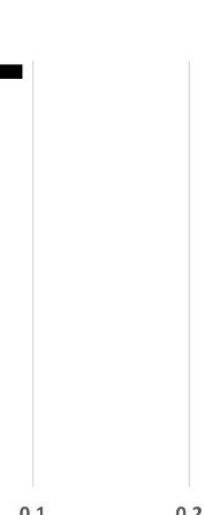

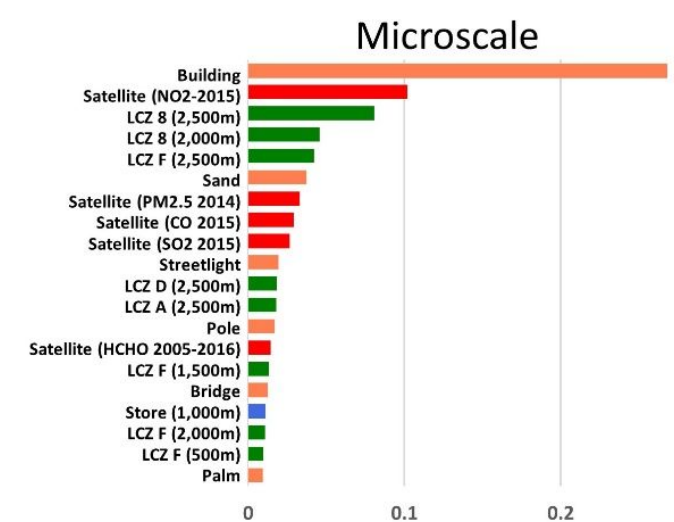

0.3

0.1

0.2

0.3
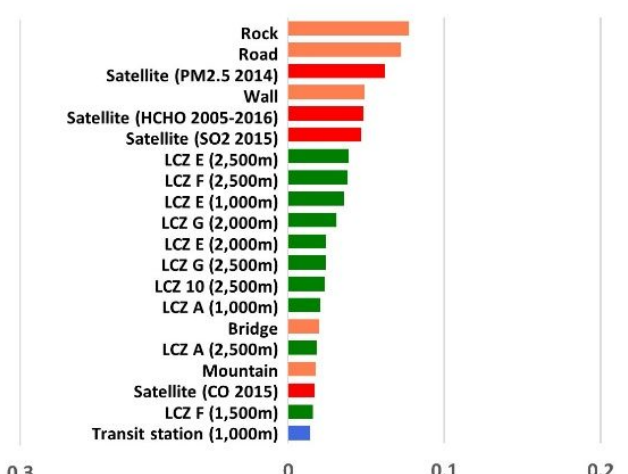
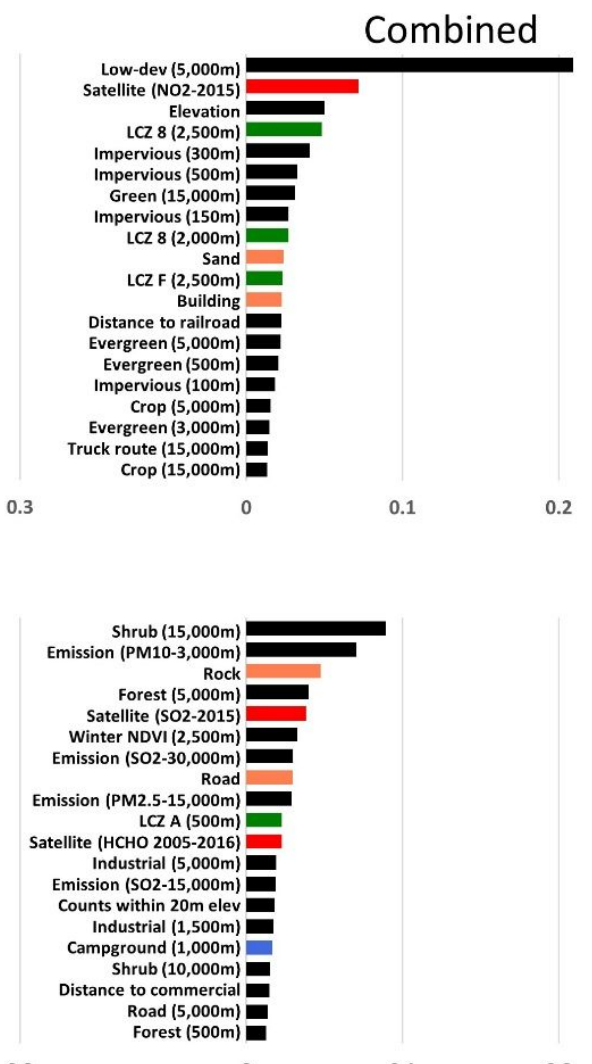

0.3

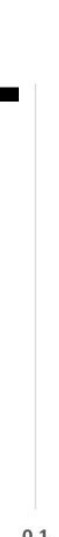

0.1

0.3

\section{Geographic}

\section{Satellite}

POI

GSV

LCZ

Figure S12. Variable importance of the top 20 variables by variable type (traditional vs. microscale. vs. combined) for the ML-based models of $\mathrm{PM}_{10}$ and $\mathrm{SO}_{2}$. For example, impervious $(5,000 \mathrm{~m})$ means area of impervious surface within $5,000 \mathrm{~m}$. Satellite $\left(\mathrm{NO}_{2}-2015\right)$ means satellite estimates of $\mathrm{NO}_{2}$ in 2015. LCZ $8(1,500 \mathrm{~m})$ means number of LCZ 8: Large low-rise grids within 1,500m. 

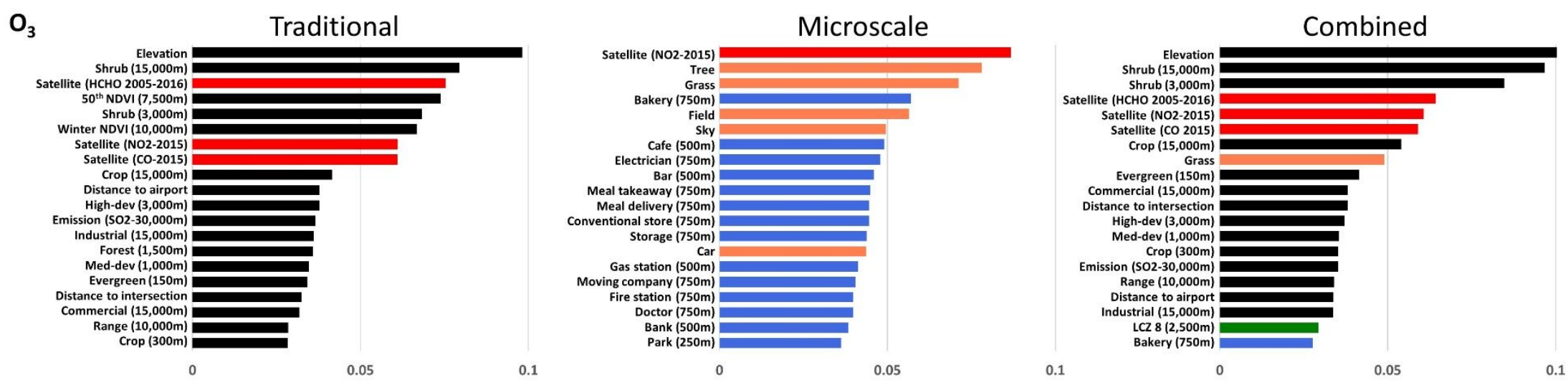

CO
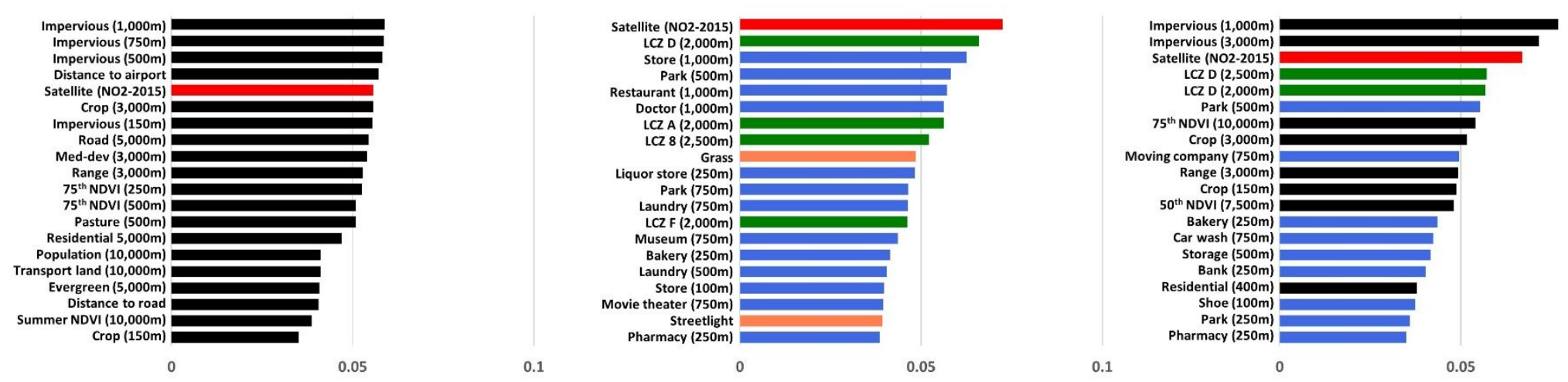

0

0.05

\section{Geographic}

Satellite

POI

GSV

LCZ

Figure S13. Variable importance of the top 20 variables by variable type (traditional vs. microscale vs. combined) for the PLS-UK models of $\mathrm{O}_{3}$ and $\mathrm{CO}$; variable importance was calculated based on the weighted VIP score (variable importance in projection). 


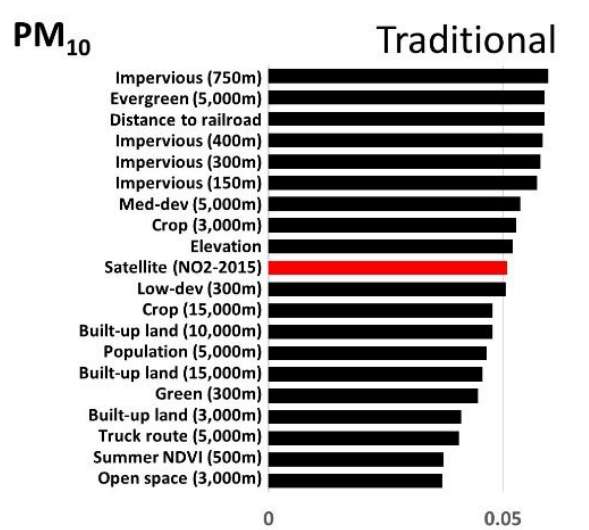

$\mathrm{SO}_{2}$

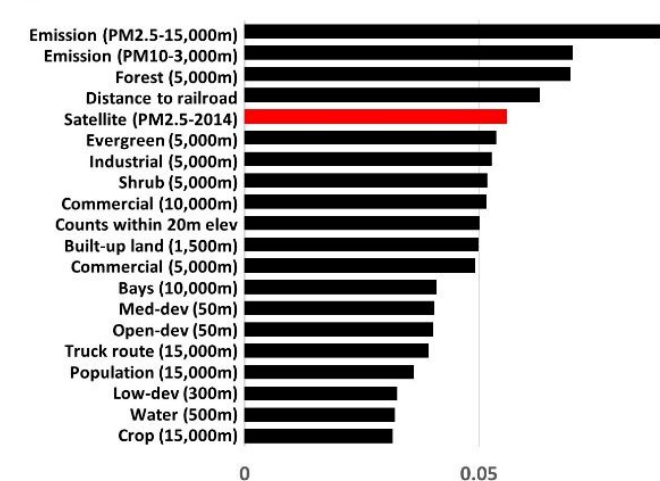

Geographic

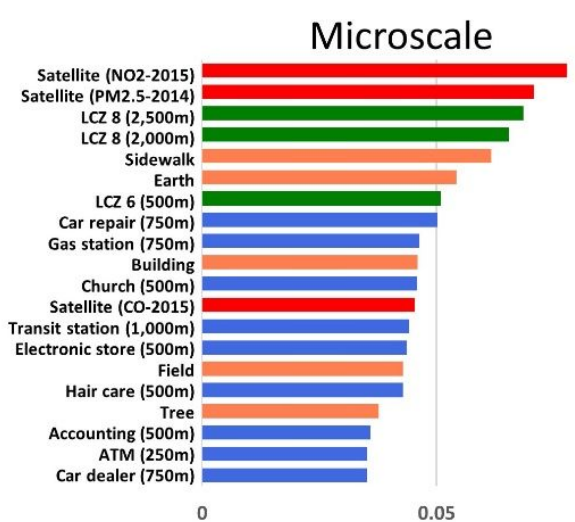

0.05

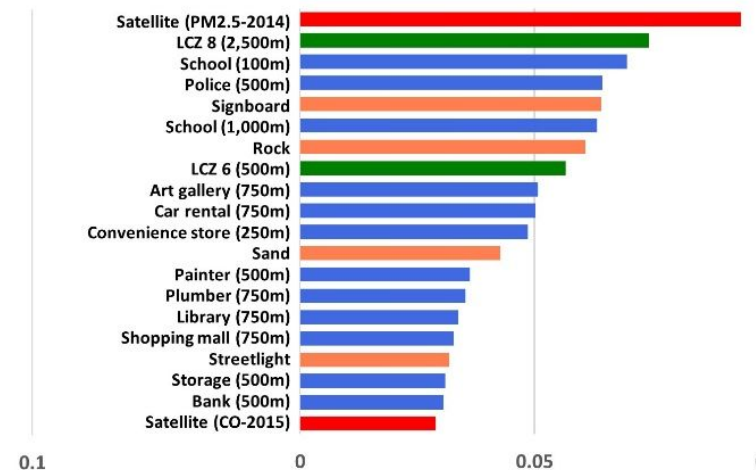

Satellite
0.05

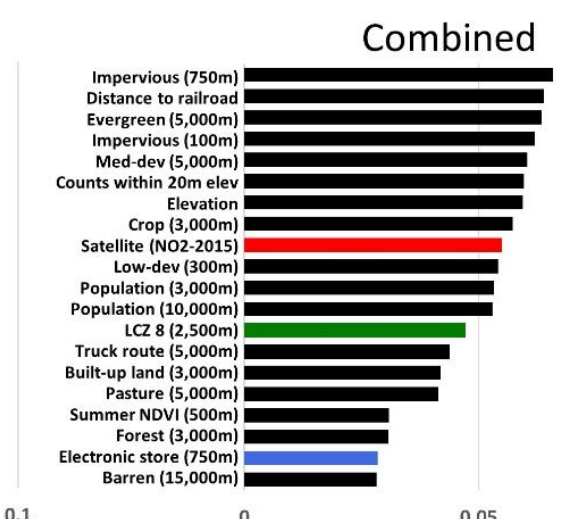

0.05

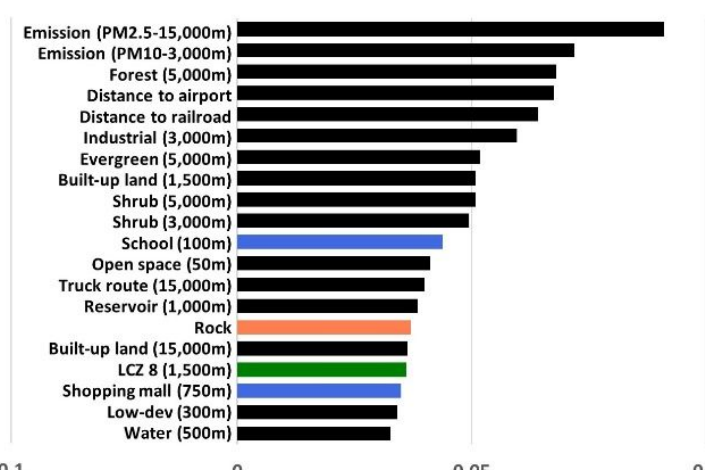

GSV

Figure S14. Variable importance of the top 20 variables by variable type (traditional vs. microscale vs. combined) for the PLS-UK models of $\mathrm{PM}_{10}$ and $\mathrm{SO}_{2}$; variable importance was calculated based on the weighted VIP score (variable importance in projection). 

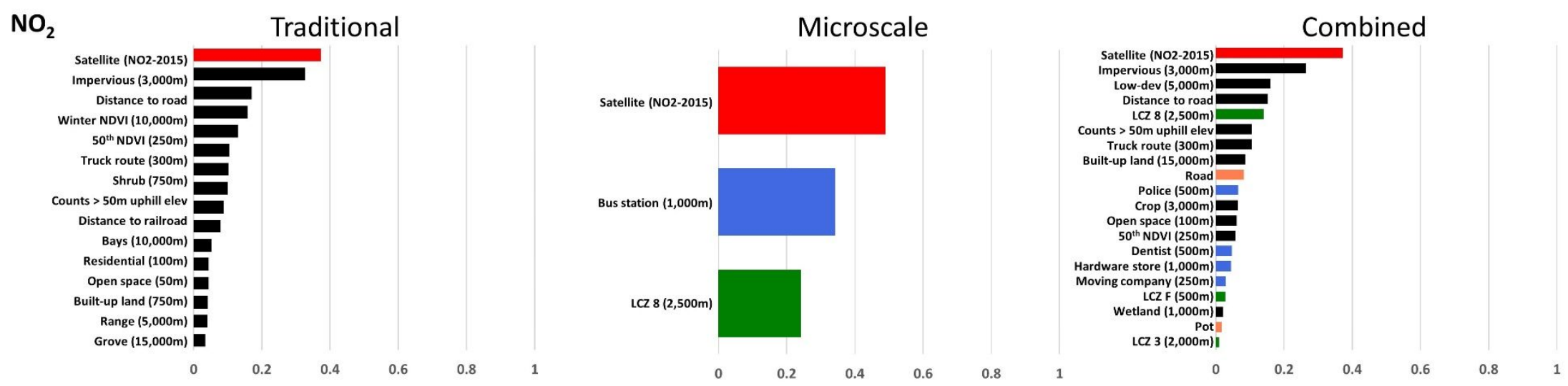

$\mathrm{PM}_{2.5}$
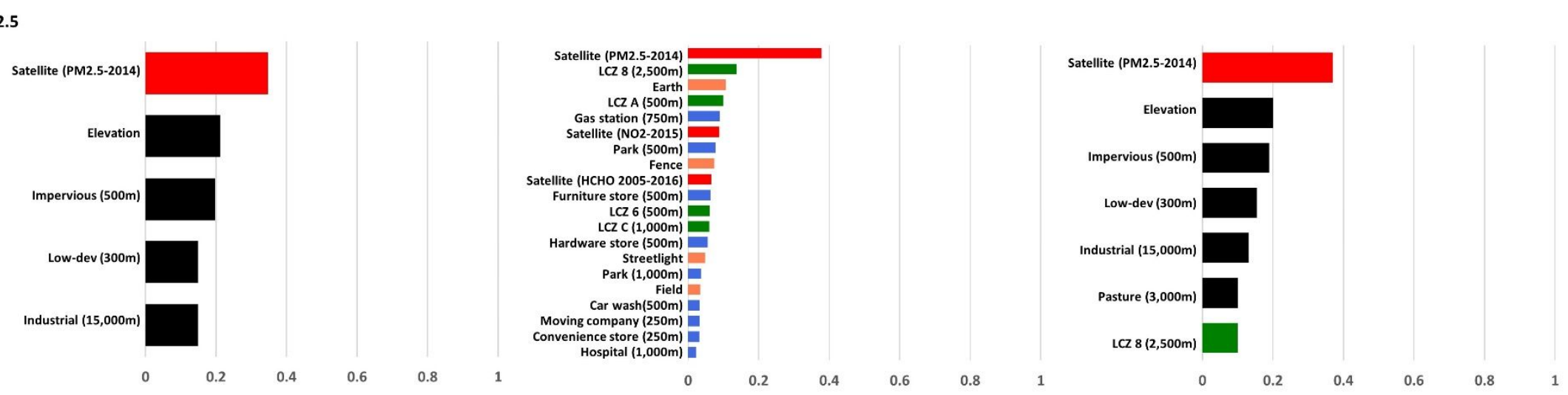

\section{Geographic}

Satellite

POI

GSV

LCZ

Figure S15. Variable importance of the top 20 variables by variable type (traditional vs. new vs. all) for the $\mathrm{SW}-\mathrm{K}$ models of $\mathrm{NO}_{2}$ and $\mathrm{PM}_{2.5}$; variable importance was represented by the normalized coefficients in the regression process during variable selection. That is, to multiple the variable coefficient by a factor that equals the difference of $95^{\text {th }}$ and $5^{\text {th }}$ percentile of the independent variable divided by the difference of $95^{\text {th }}$ and $5^{\text {th }}$ percentile of the dependent variable. 

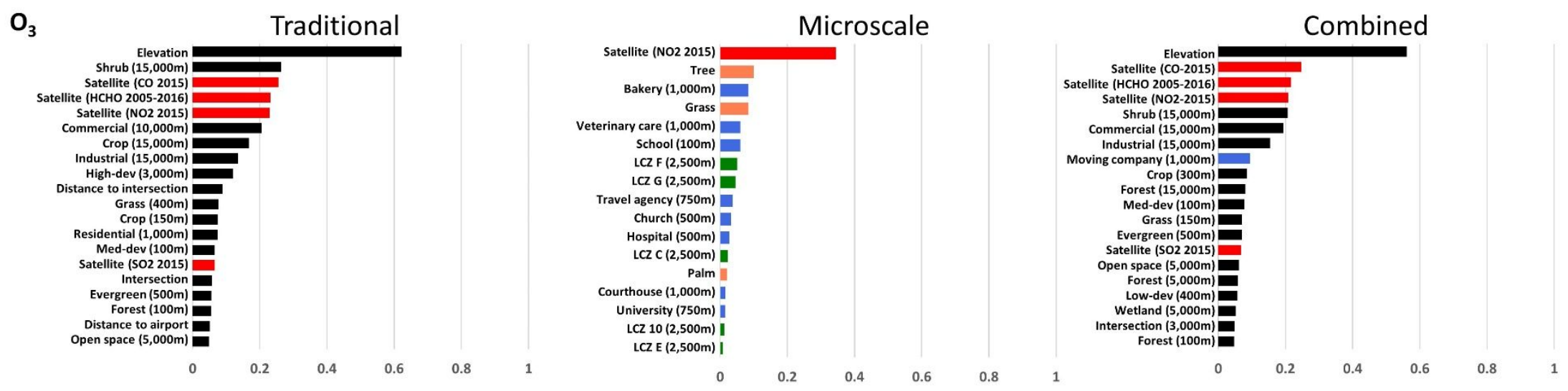

co
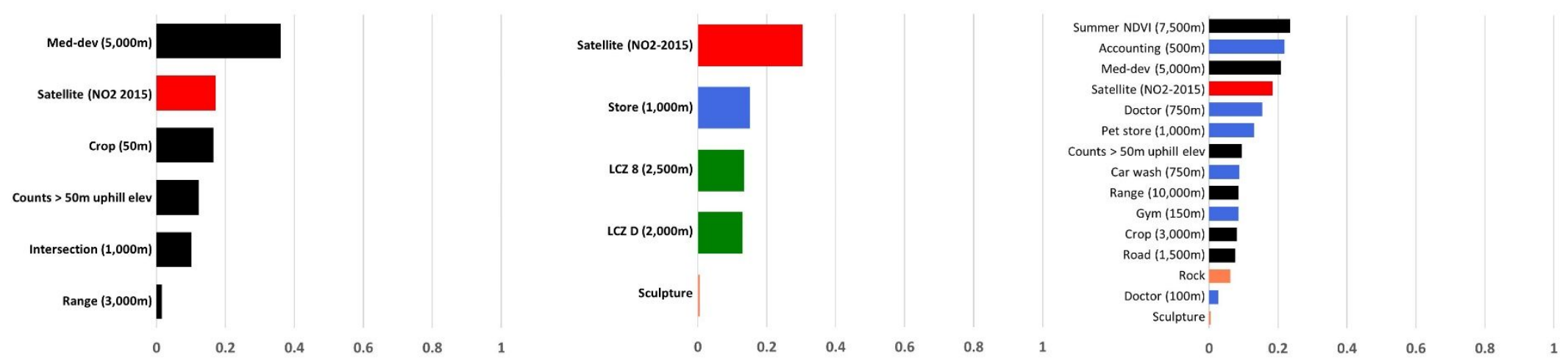

\section{Geographic}

Satellite

POI

GSV

LCZ

Figure S16. Variable importance of the top 20 variables by variable type (traditional vs. new vs. all) for the $\mathrm{SW}-\mathrm{K}$ models of $\mathrm{O}_{3}$ and $\mathrm{CO}$; variable importance was represented by the normalized coefficients in the regression process during variable selection. That is, to multiple the variable coefficient by a factor that equals the difference of $95^{\text {th }}$ and $5^{\text {th }}$ percentile of the independent variable divided by the difference of $95^{\text {th }}$ and $5^{\text {th }}$ percentile of the dependent variable. 

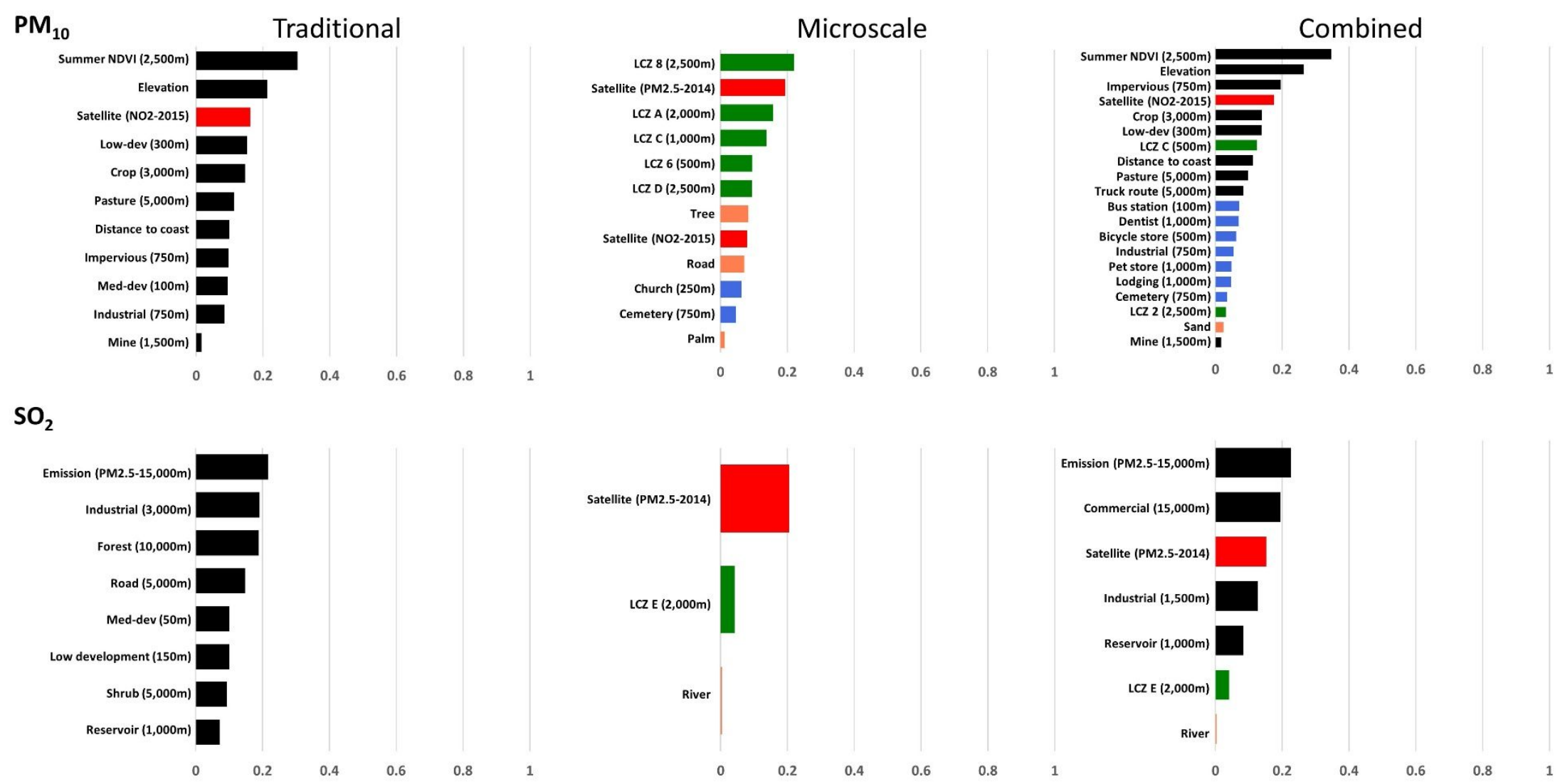

\section{Geographic \\ Satellite \\ POI \\ GSV}

LCZ

Figure S17. Variable importance of the top 20 variables by variable type (traditional vs. new vs. all) for the SW-K models of PM 10 and $\mathrm{SO}_{2}$; variable importance was represented by the normalized coefficients in the regression process during variable selection. That is, to multiple the variable coefficient by a factor that equals the difference of $95^{\text {th }}$ and $5^{\text {th }}$ percentile of the independent variable divided by the difference of $95^{\text {th }}$ and $5^{\text {th }}$ percentile of the dependent variable. 

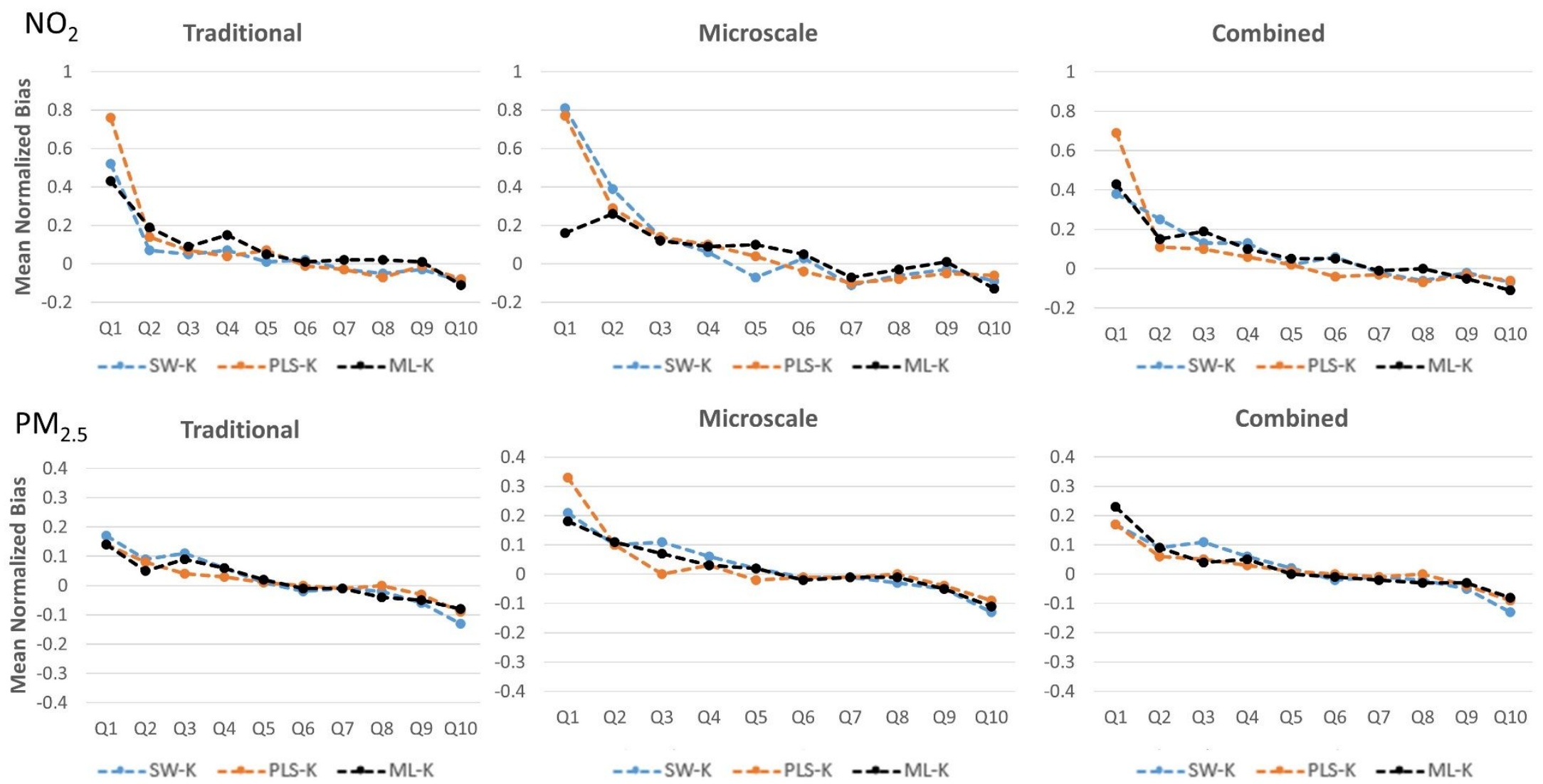

Figure S18. Random 10-fold CV results divided by 10 quantiles of $\mathrm{NO}_{2}$ and $\mathrm{PM}_{2.5}$. Q1 to Q10 indicates quantile 1 to quantile 10 of the air pollution concentrations. 

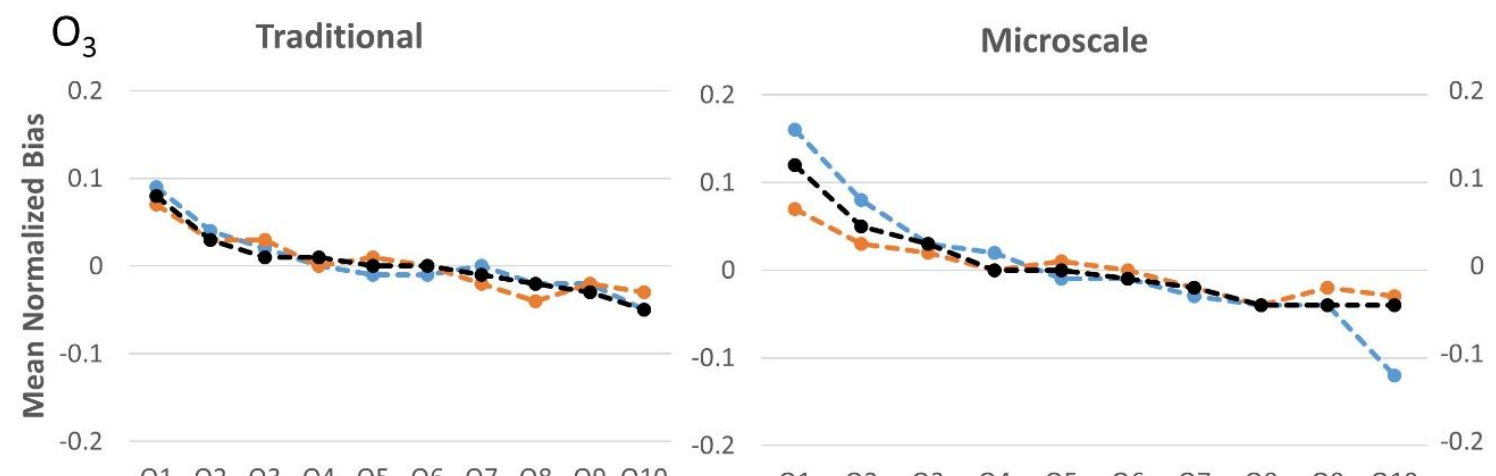

Combined

Q1 $\begin{array}{llllllllll}\text { Q2 } & \text { Q3 } & \text { Q4 } & \text { Q5 } & \text { Q6 } & \text { Q7 } & \text { Q8 } & \text { Q9 } & \text { Q10 }\end{array}$

$\begin{array}{llllllllll}\text { Q1 } & \text { Q2 } & \text { Q3 } & \text { Q4 } & \text { Q5 } & \text { Q6 } & \text { Q7 } & \text { Q8 } & \text { Q9 } & \text { Q10 }\end{array}$

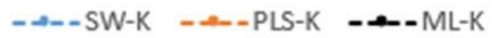

CO Traditional

Microscale
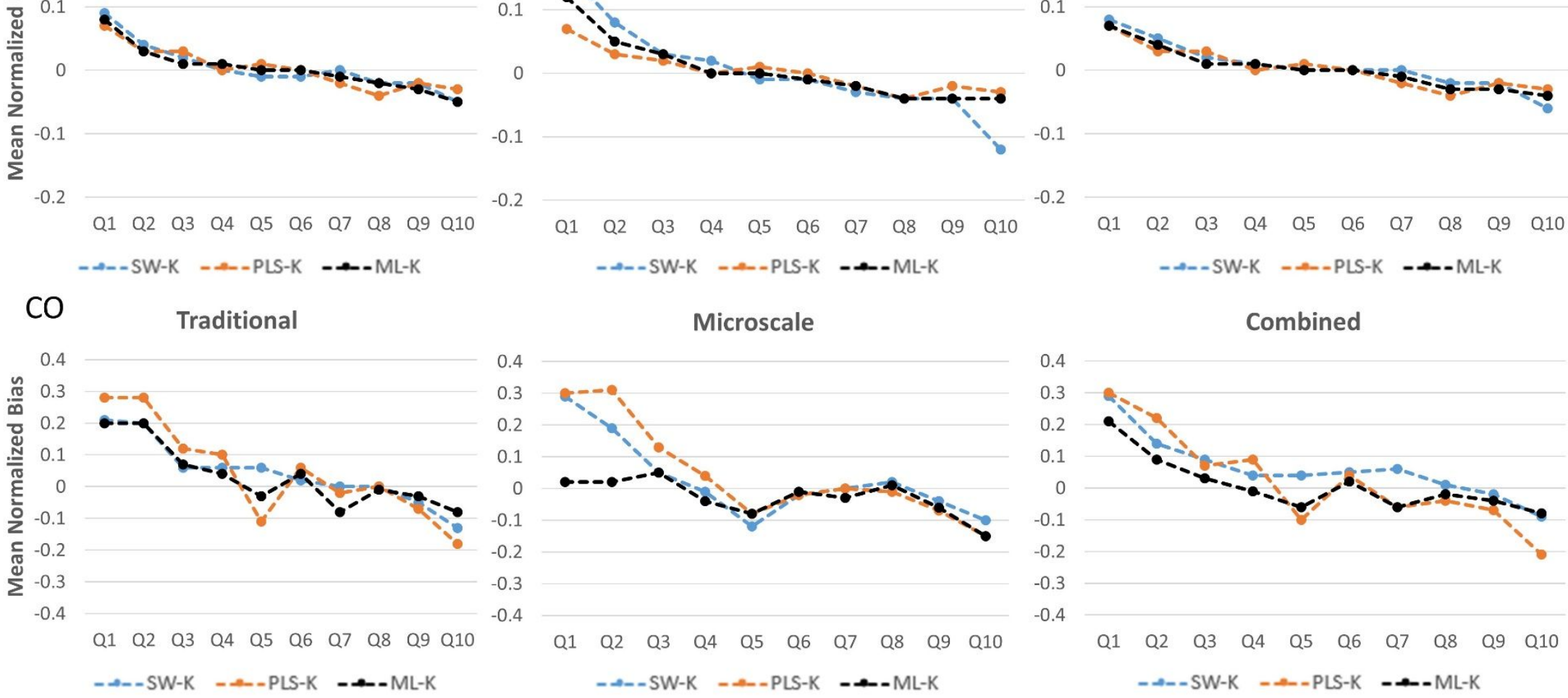

Figure S19. Random 10-fold CV results divided by 10 quantiles of $\mathrm{O}_{3}$ and CO. Q1 to Q10 indicates quantile 1 to quantile 10 of the air pollution concentrations. 


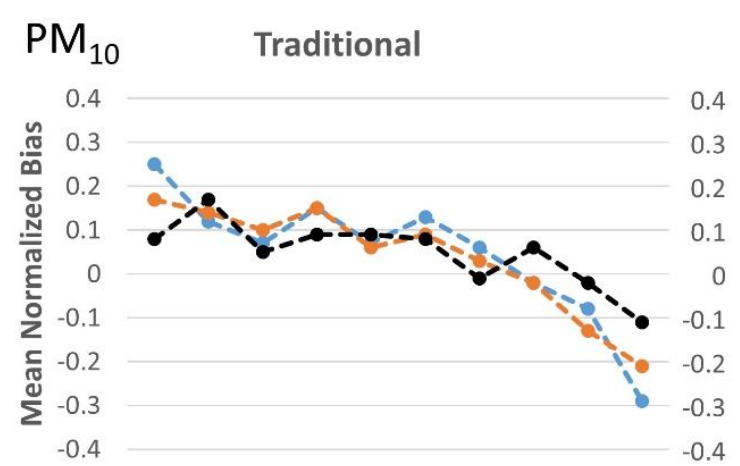

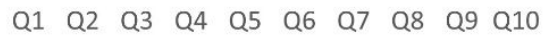
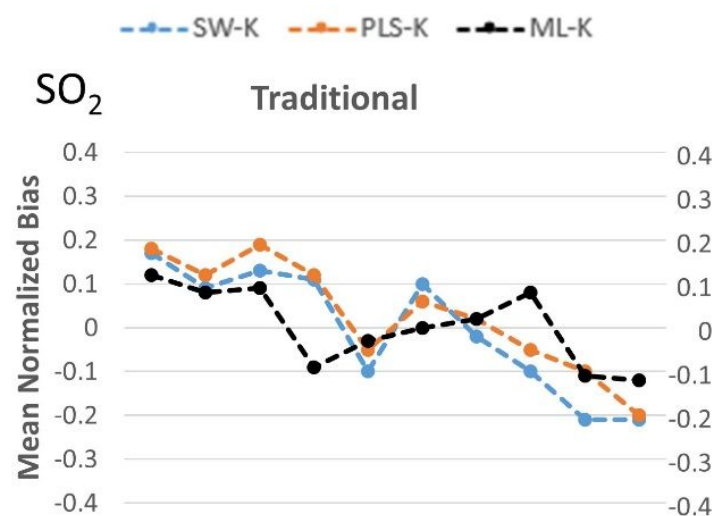

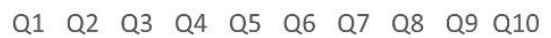

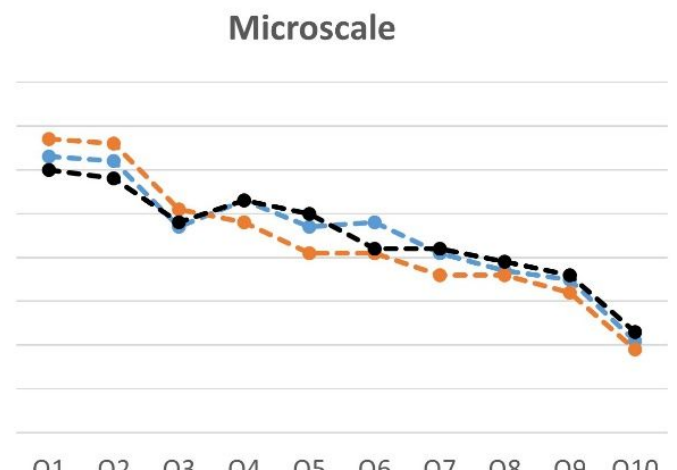

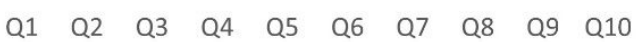

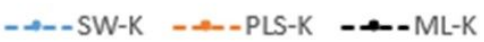

Microscale

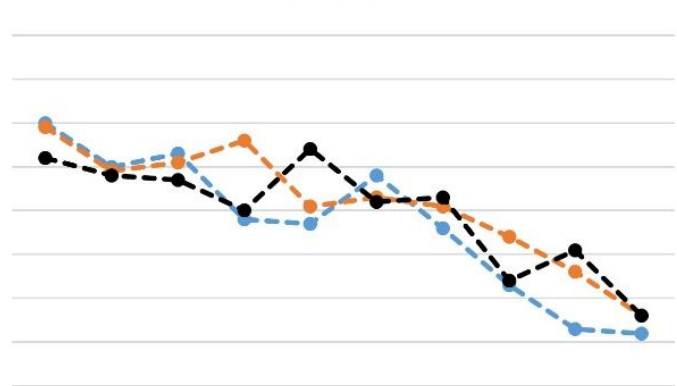

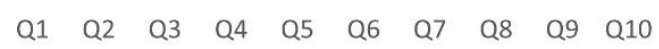

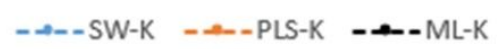

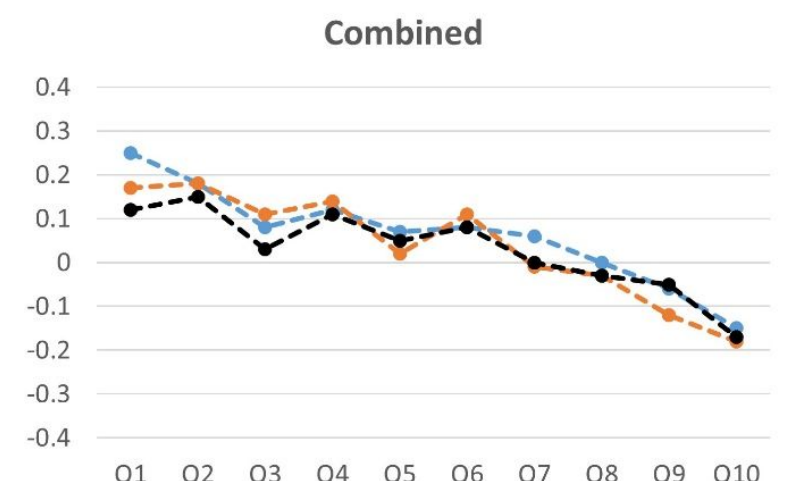

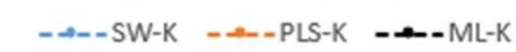

Combined

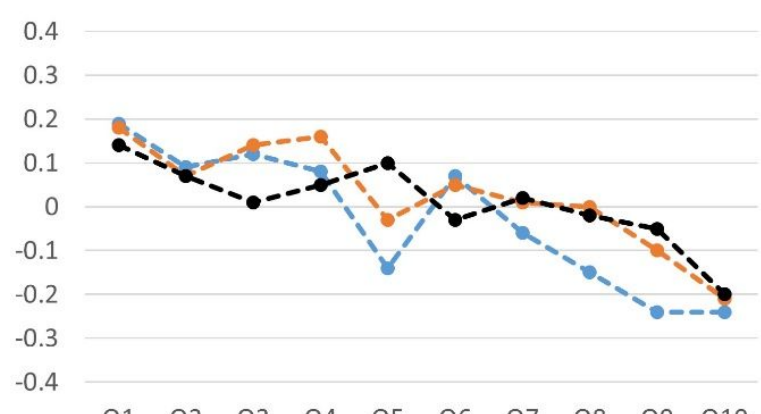

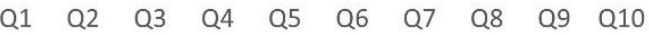
- -- SW-K $\quad-\rightarrow-P L S-K \quad--M L-K$

Figure S20. Random 10-fold CV results divided by 10 quantiles of $\mathrm{PM}_{10}$ and $\mathrm{SO}_{2}$. Q1 to Q10 indicates quantile 1 to quantile 10 of the air pollution concentrations. 

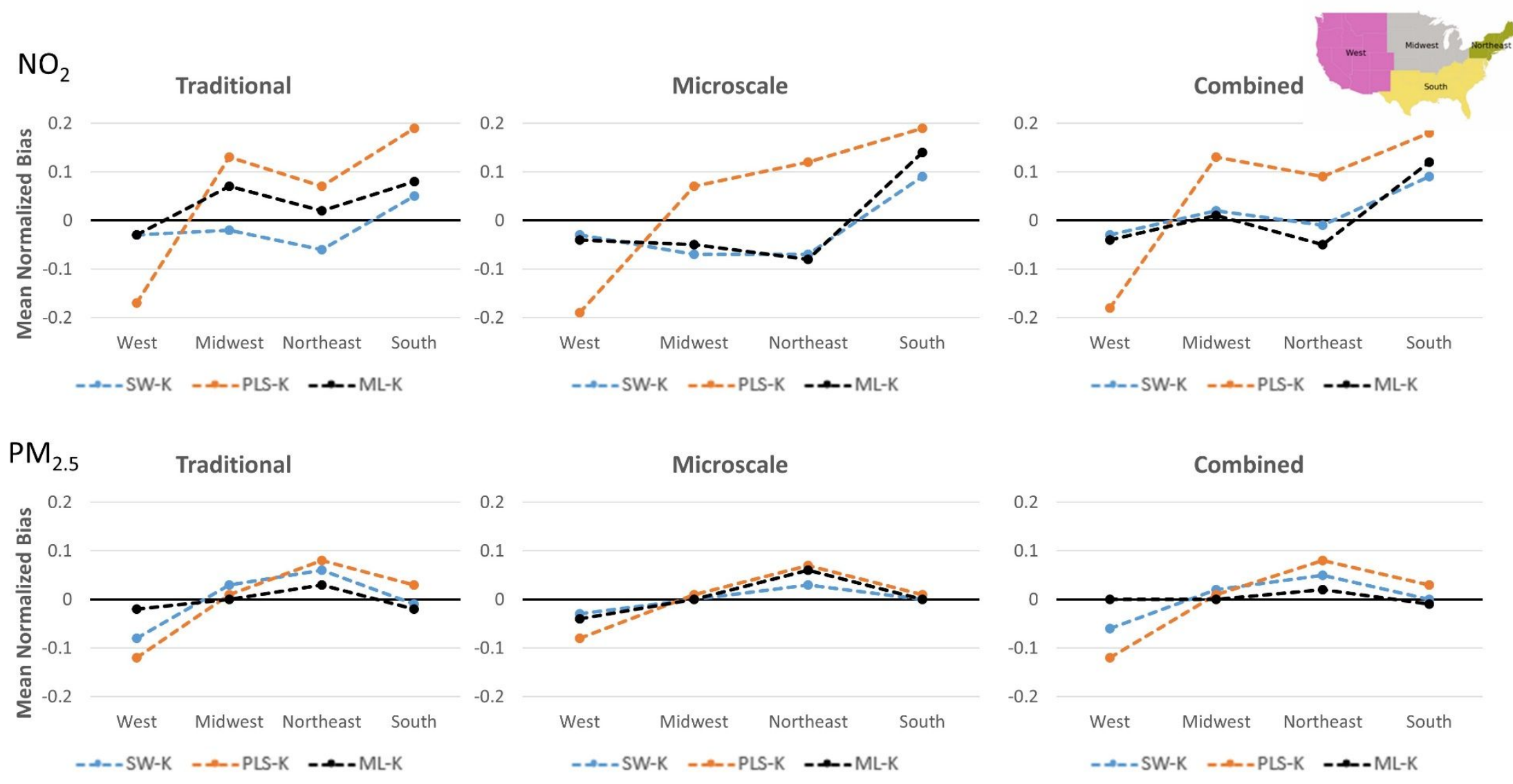

Figure S21. Random 10-fold CV results based on monitoring locations according to the four Census Bureau-designated regions (i.e., West, Midwest, Northeast, and South) for $\mathrm{NO}_{2}$ and $\mathrm{PM}_{2.5}$. 


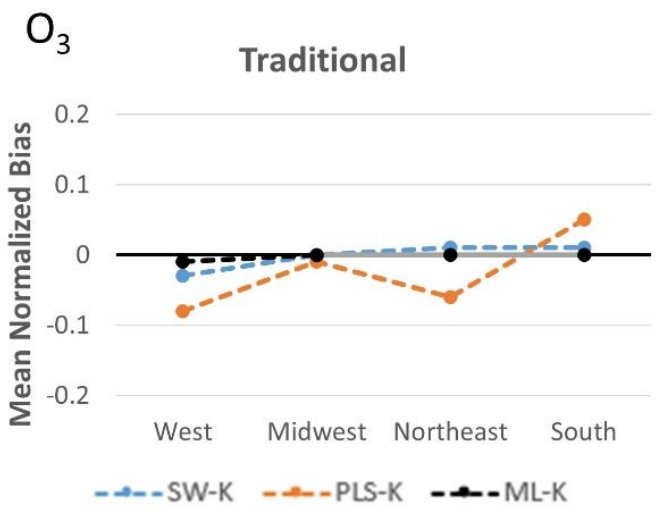

Microscale

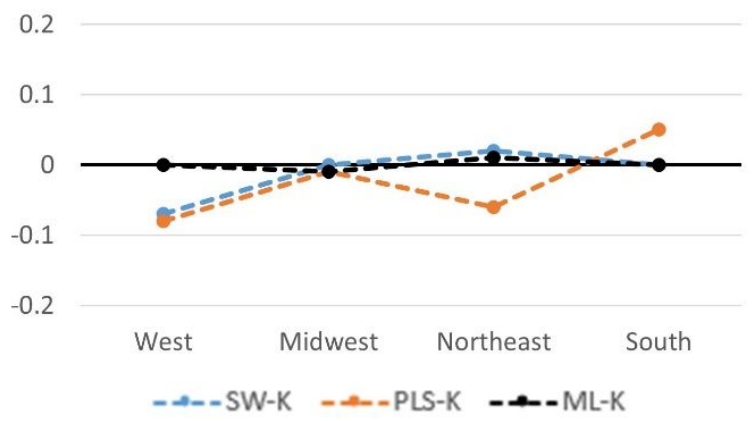

$\mathrm{CO}$

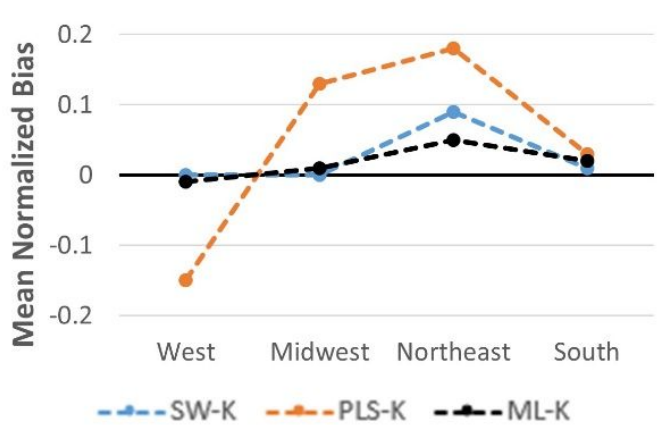

Microscale

0.2

0.1
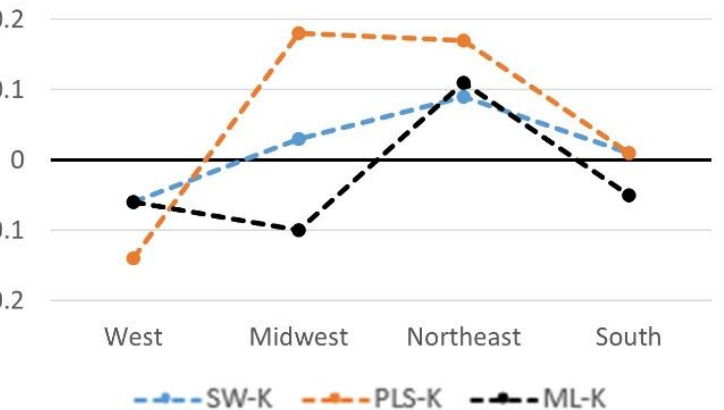

Combined

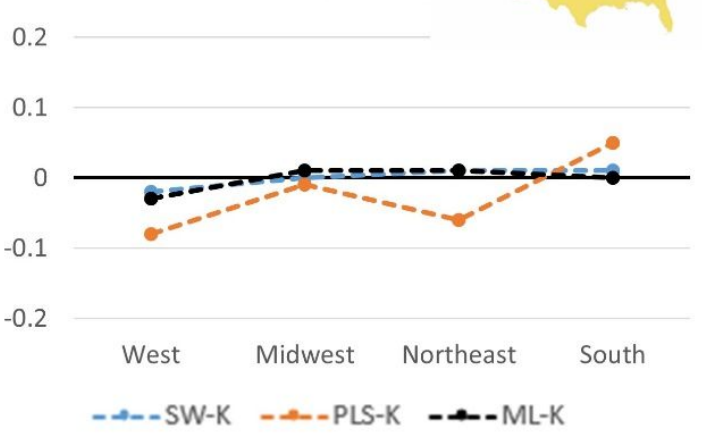

Combined

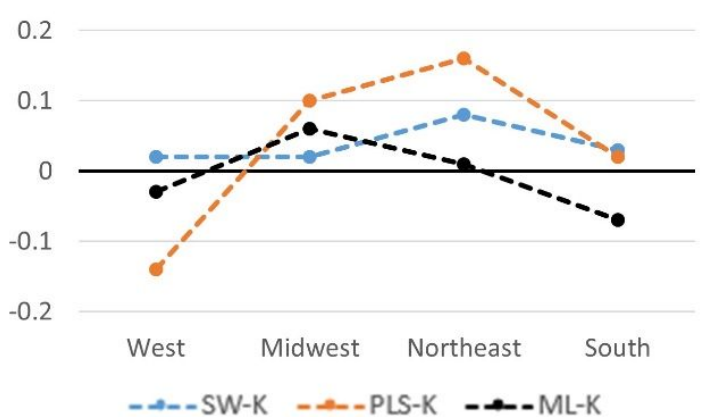

Figure S22. Random 10-fold CV results based on monitoring locations according to the four Census Bureau-designated regions (i.e., West, Midwest, Northeast, and South) for $\mathrm{O}_{3}$ and $\mathrm{CO}$. 

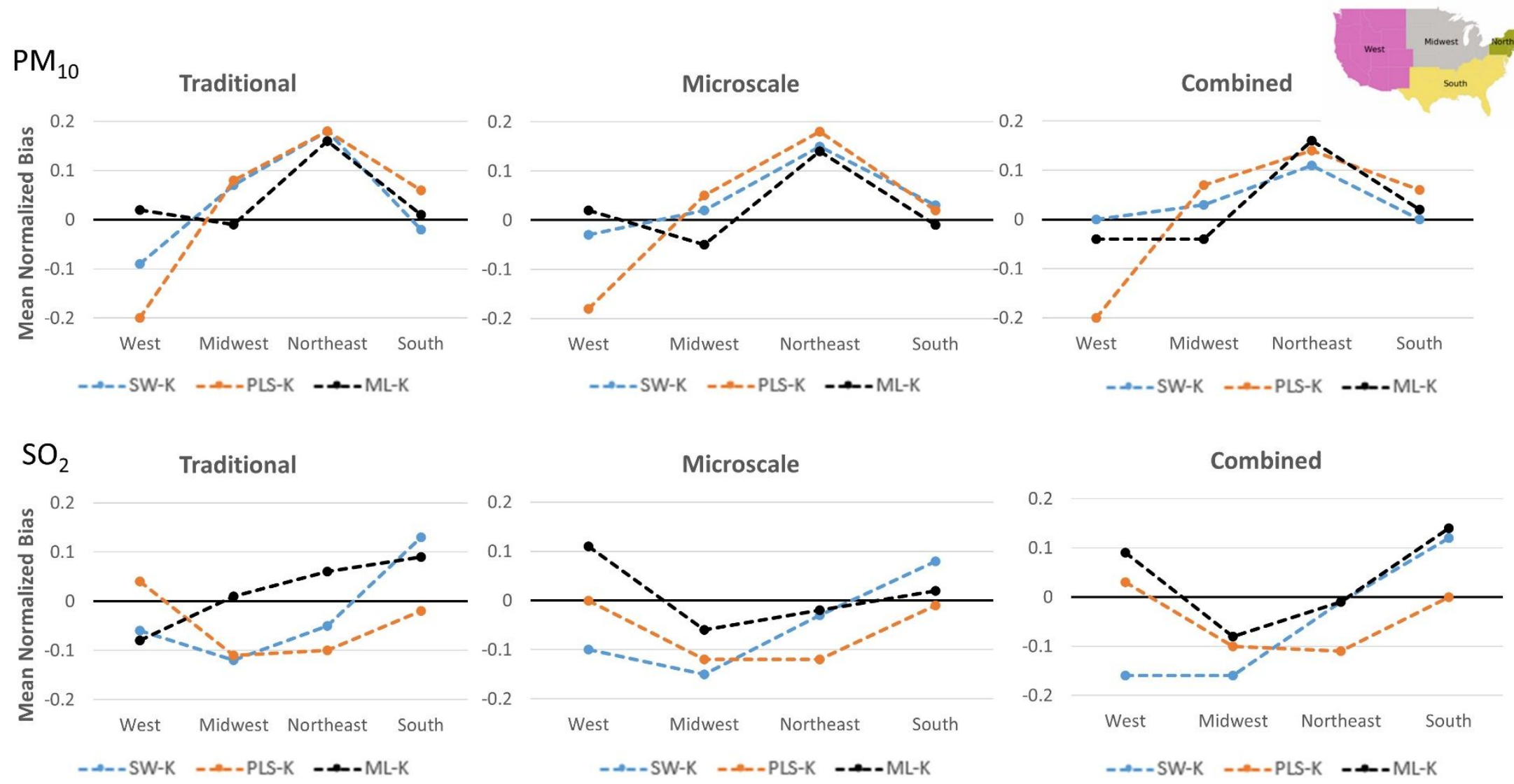

Figure S23. Random 10-fold CV results based on monitoring locations according to the four Census Bureau-designated regions (i.e., West, Midwest, Northeast, and South) for $\mathrm{PM}_{10}$ and $\mathrm{SO}_{2}$. 

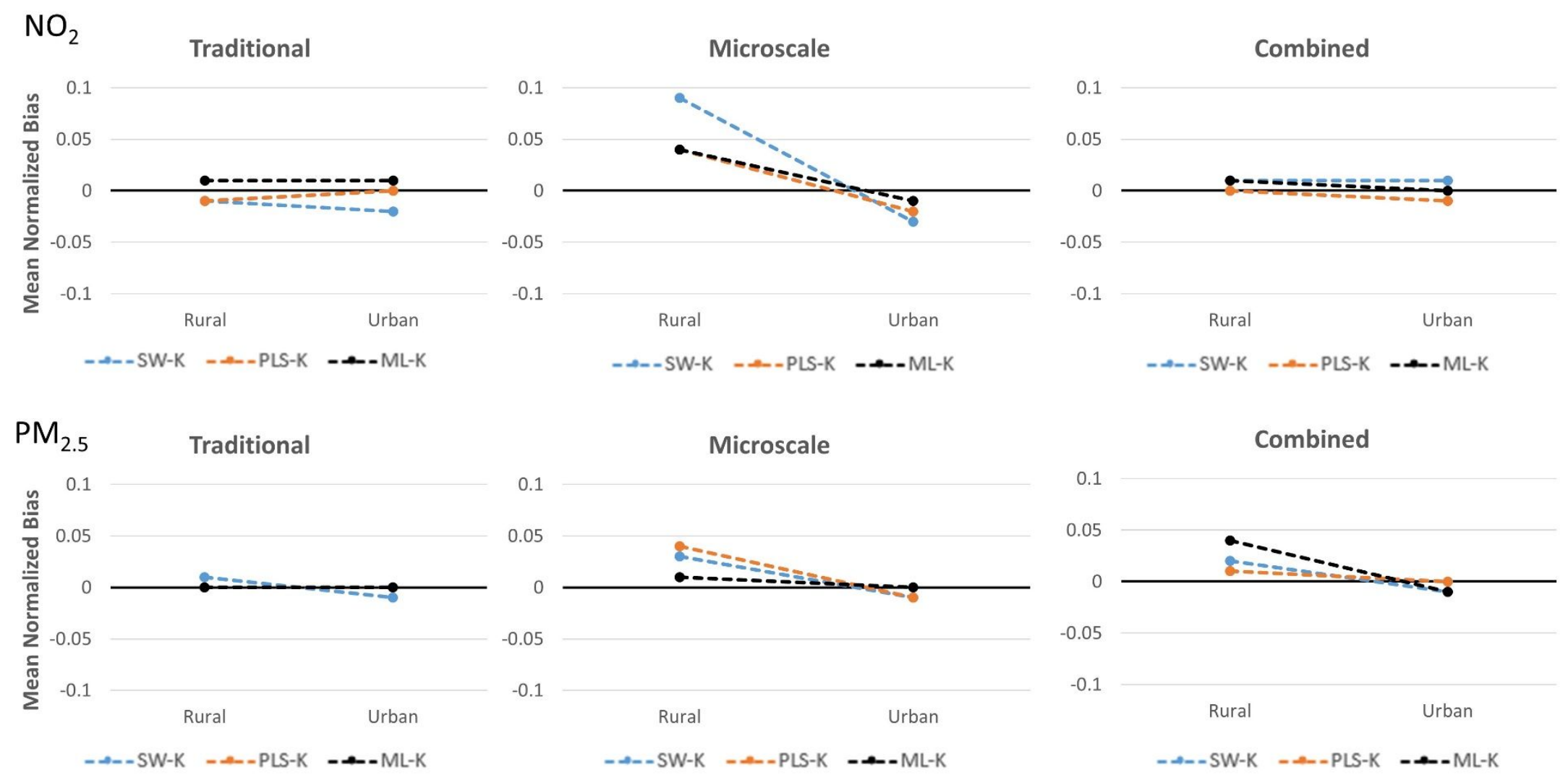

Figure S24. Random 10-fold CV results based on monitoring locations in urban and rural areas according to the US Census classification for $\mathrm{NO}_{2}$ and $\mathrm{PM}_{2.5}$. 

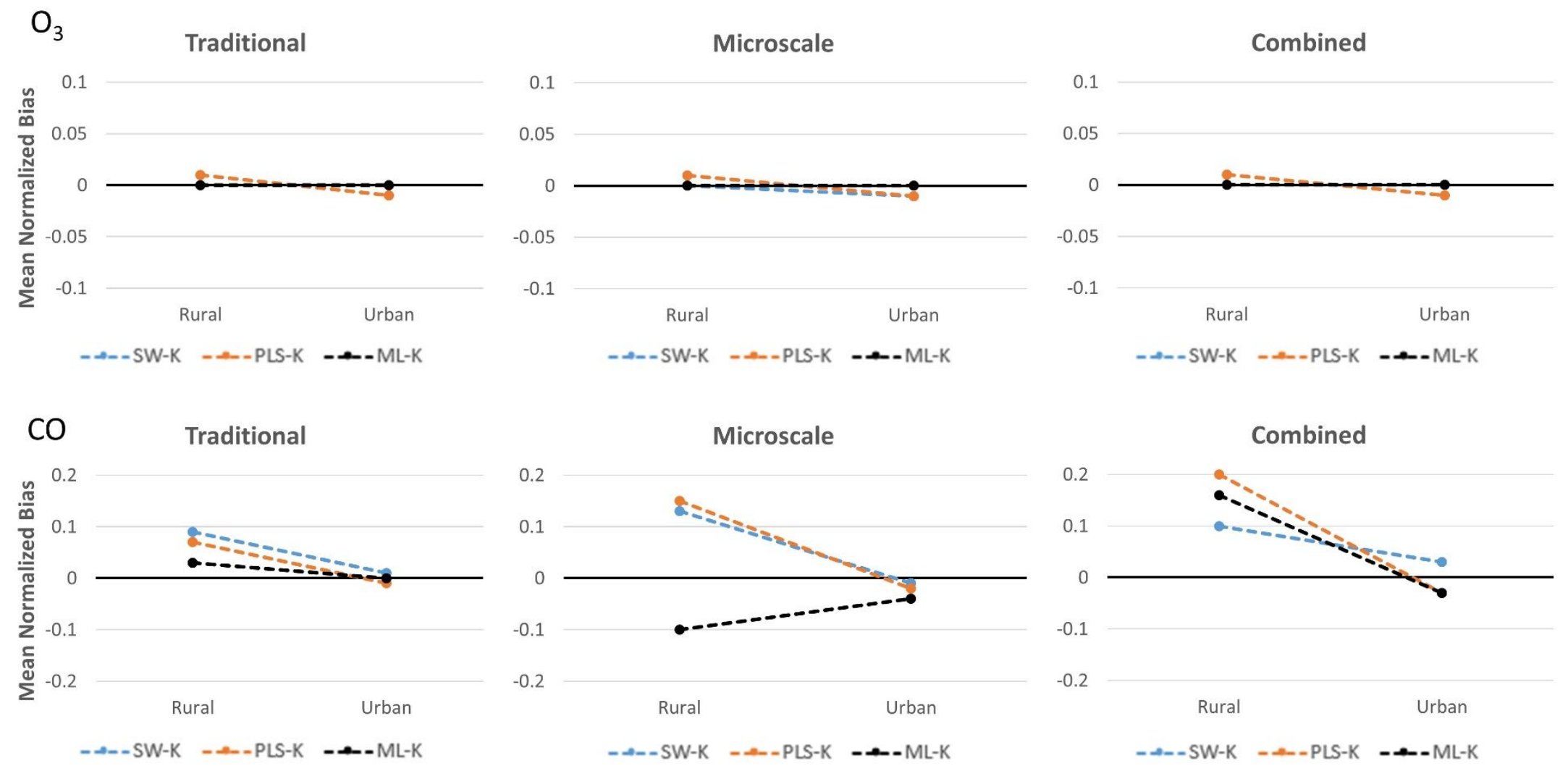

Figure S25. Random 10-fold CV results based on monitoring locations in urban and rural areas according to the US Census classification for $\mathrm{O}_{3}$ and $\mathrm{CO}$. 

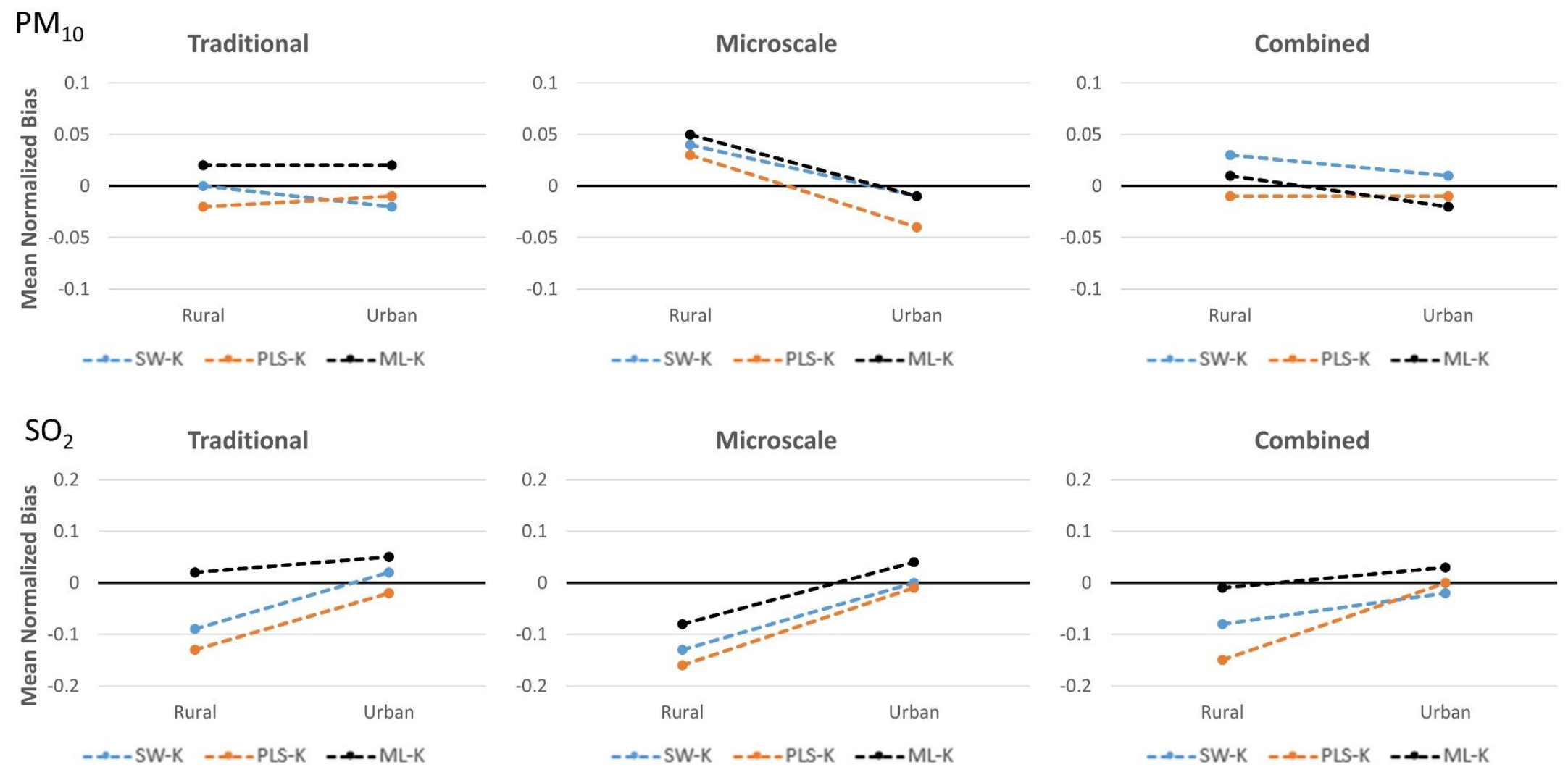

Figure S26. Random 10-fold CV results based on monitoring locations in urban and rural areas according to the US Census classification for $\mathrm{PM}_{10}$ and $\mathrm{SO}_{2}$. 

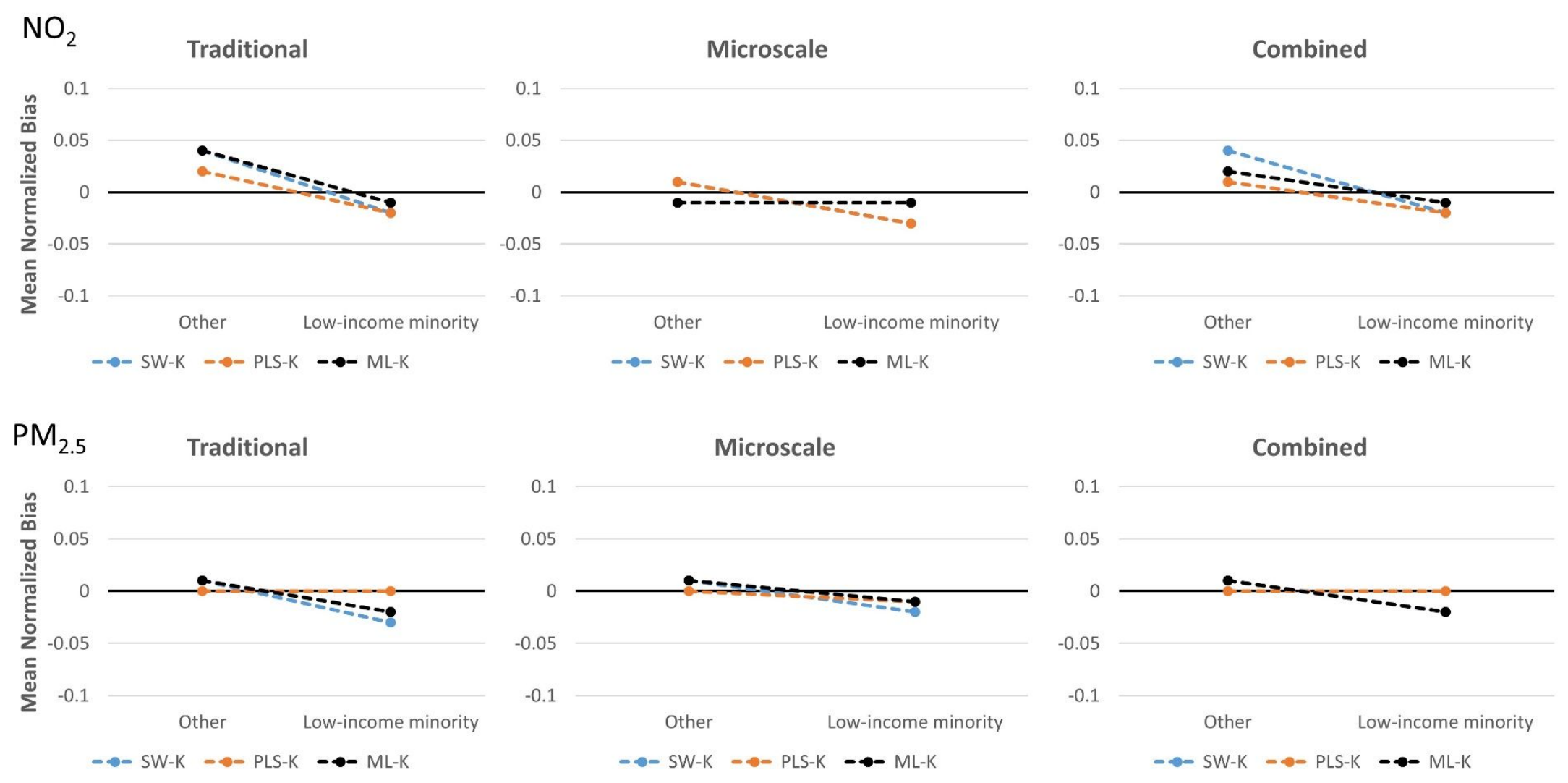

Figure S27. Random 10-fold CV results based on monitoring locations in low-income minority area and other area according to the demographic index of EJSCREEN for $\mathrm{NO}_{2}$ and $\mathrm{PM}_{2.5}$. 

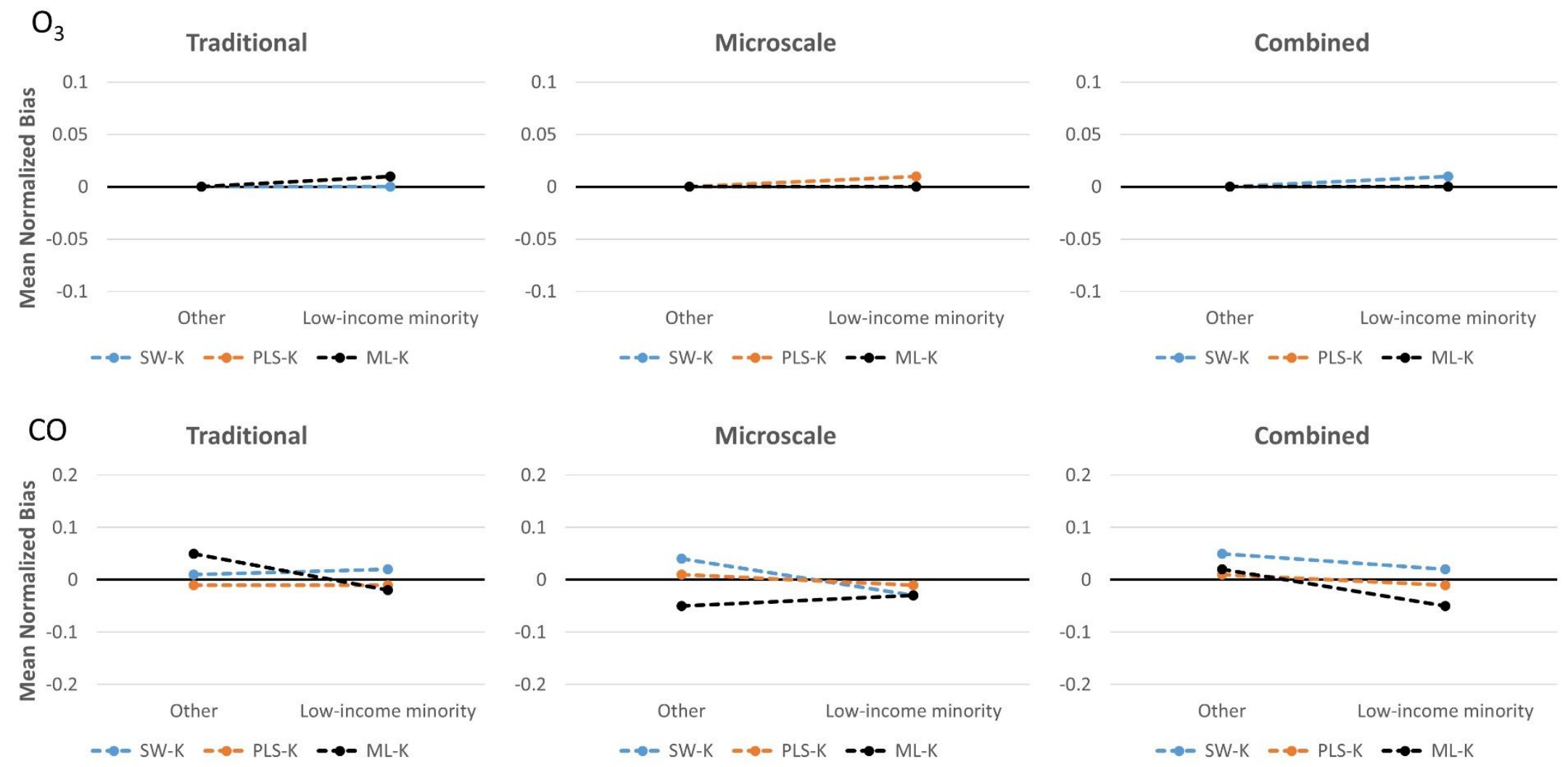

Figure S28. Random 10-fold CV results based on monitoring locations in low-income minority area and other area according to the demographic index of EJSCREEN for $\mathrm{O}_{3}$ and $\mathrm{CO}$. 

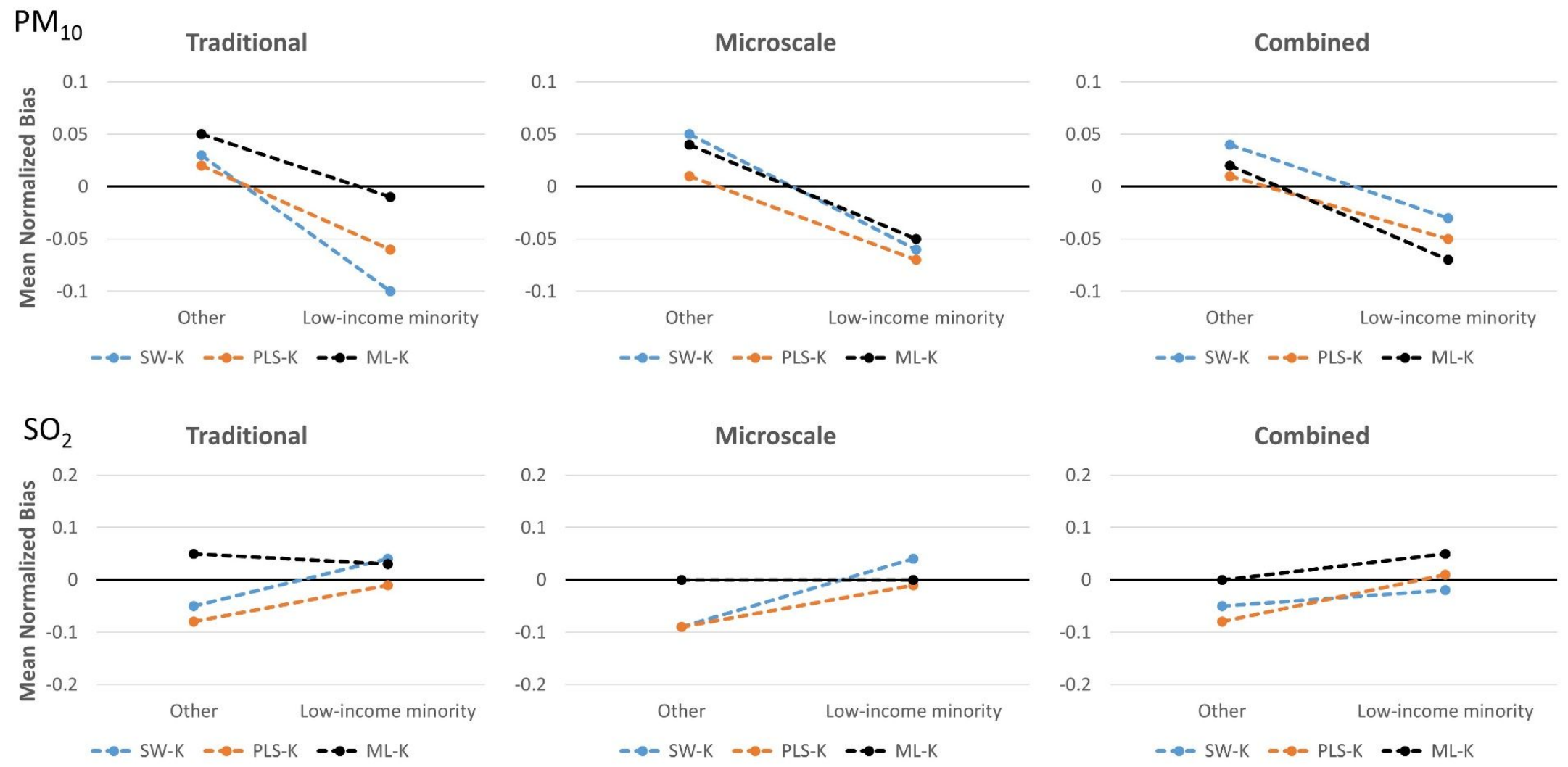

Figure S29. Random 10-fold CV results based on monitoring locations in low-income minority area and other area according to the demographic index of EJSCREEN for $\mathrm{PM}_{10}$ and $\mathrm{SO}_{2}$. 


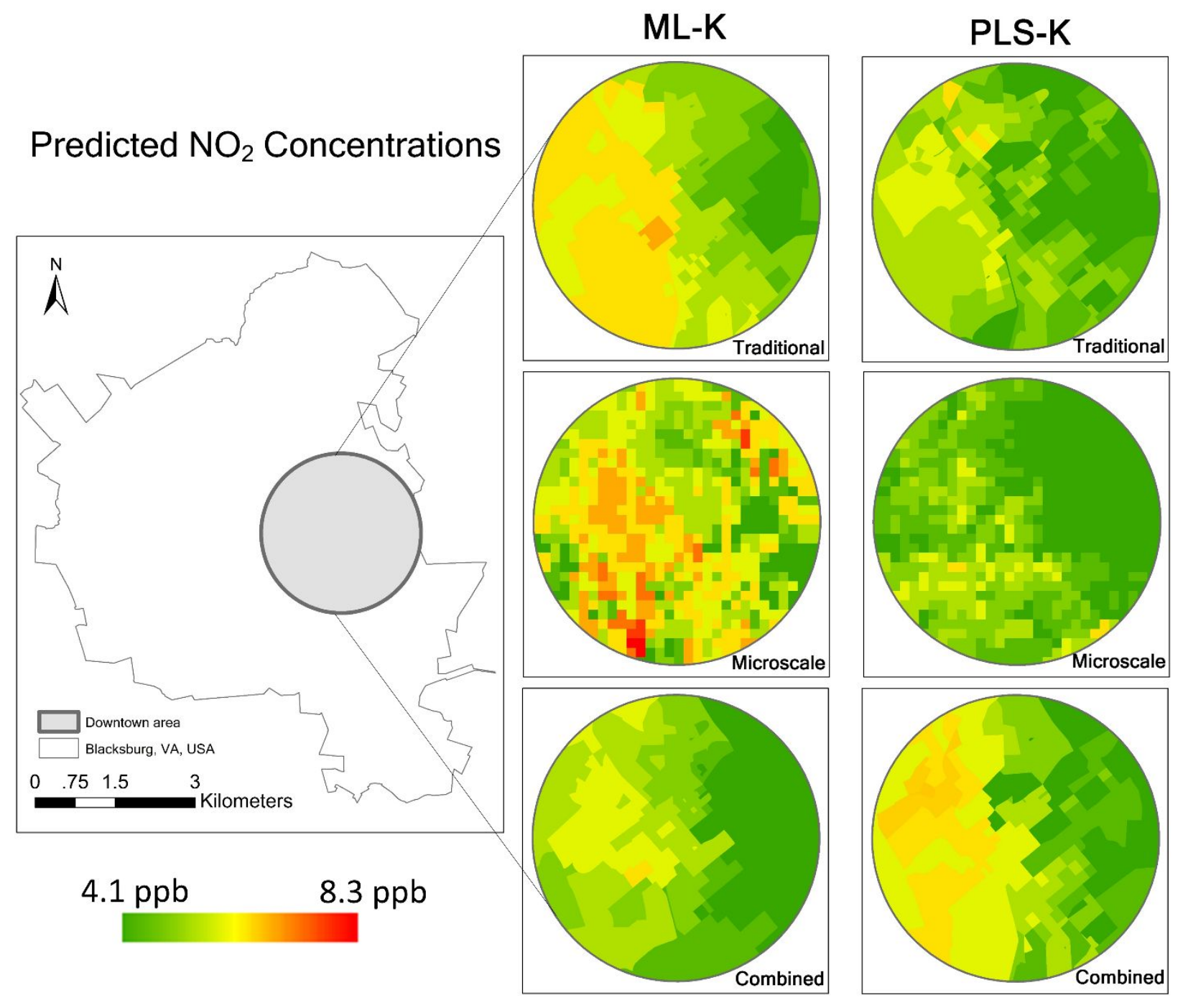

Figure 30. Predicted $\mathrm{NO}_{2}$ concentrations using ML-K and PLS-K models for downtown Blacksburg, VA. Traditional and combined models were developed at the census block level while microscale models were developed at $100 \mathrm{~m}$ spatial resolution due to data availability. 


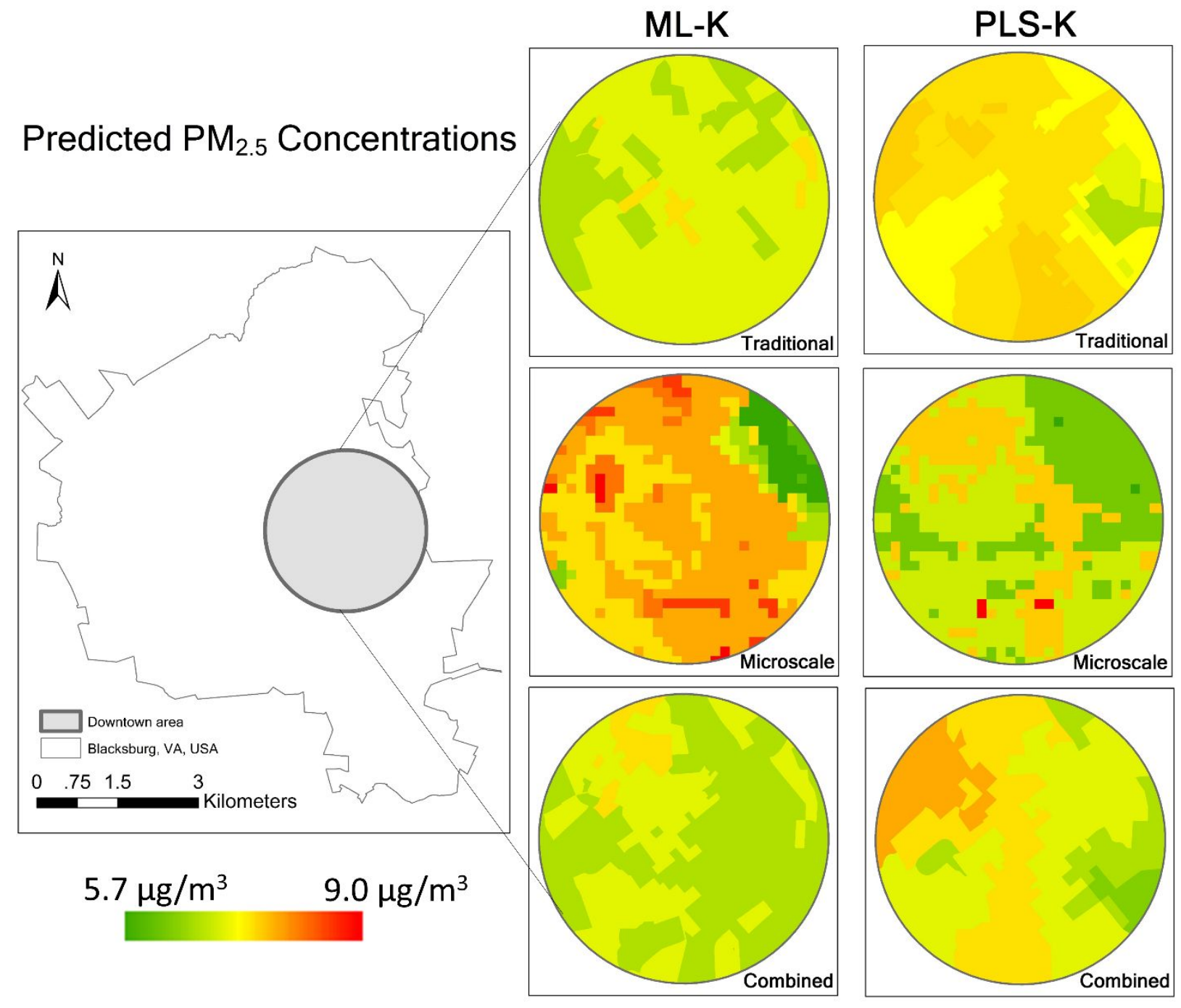

Figure 31. Predicted $\mathrm{PM}_{2.5}$ concentrations using ML-K and PLS-K models for downtown Blacksburg, VA. Traditional and combined models were developed at the census block level while microscale models were developed at $100 \mathrm{~m}$ spatial resolution due to data availability. 


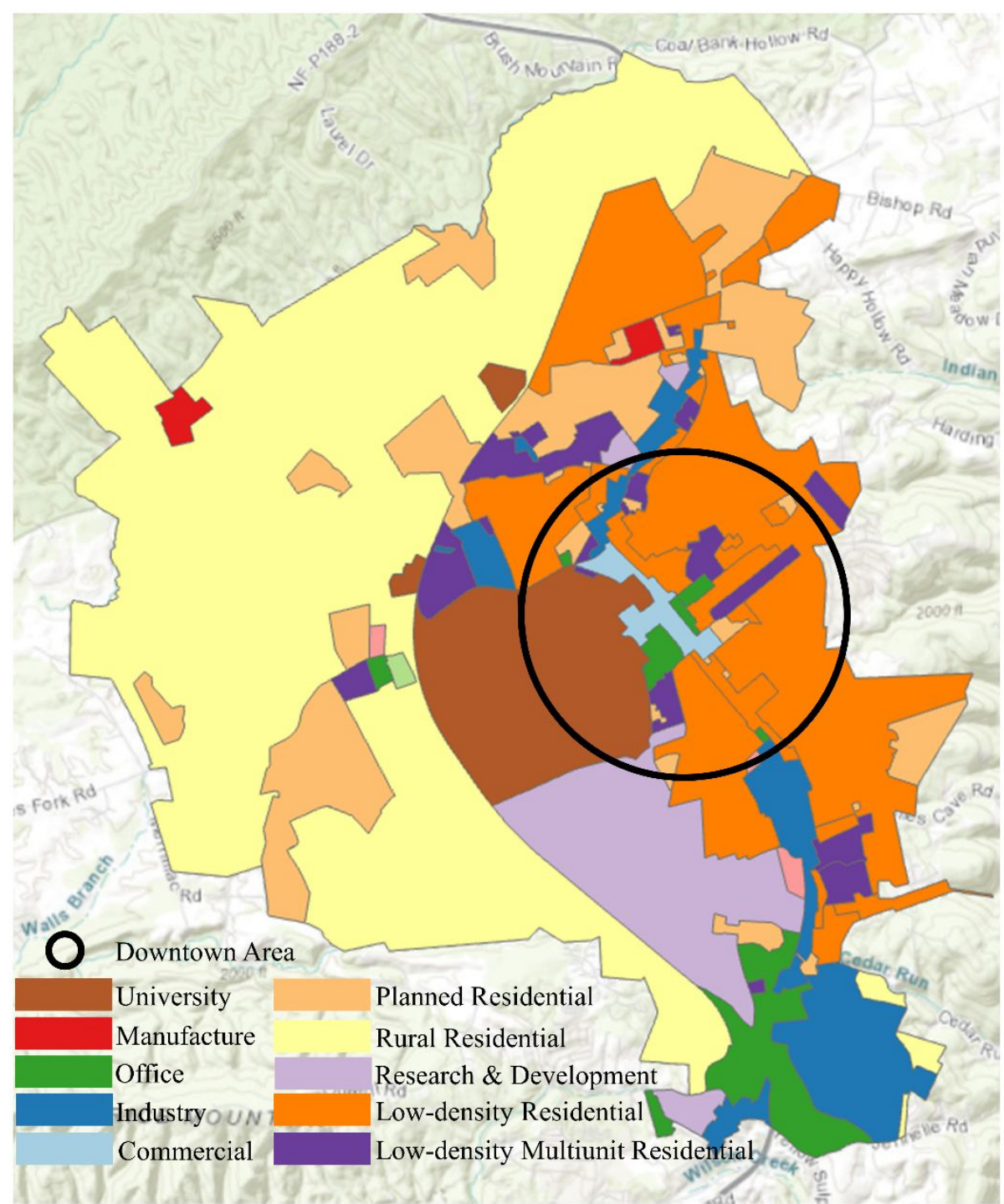

Figure S32. The zoning map of Blacksburg, VA, USA. 